\title{
Fast Optical Transients from Stellar-mass Black Hole Tidal Disruption Events in Young Star Clusters
}

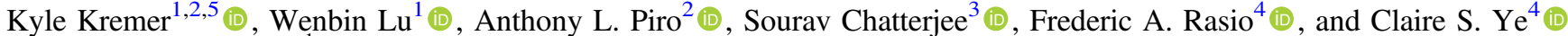 \\ ${ }^{1}$ TAPIR, California Institute of Technology, Pasadena, CA 91125, USA; kkremer@caltech.edu \\ ${ }^{2}$ The Observatories of the Carnegie Institution for Science, Pasadena, CA 91101, USA \\ ${ }^{3}$ Tata Institute of Fundamental Research, Homi Bhabha Road, Navy Nagar, Colaba, Mumbai 400005, India \\ ${ }^{4}$ Center for Interdisciplinary Exploration \& Research in Astrophysics (CIERA) and Department of Physics \& Astronomy, Northwestern University, Evanston, IL \\ 60208, USA \\ Received 2020 December 3; revised 2021 February 19; accepted 2021 February 27; published 2021 April 22
}

\begin{abstract}
Observational evidence suggests that the majority of stars may have been born in stellar clusters or associations. Within these dense environments, dynamical interactions lead to high rates of close stellar encounters. A variety of recent observational and theoretical indications suggest stellar-mass black holes may be present and play an active dynamical role in stellar clusters of all masses. In this study, we explore the tidal disruption of main-sequence stars by stellar-mass black holes in young star clusters. We compute a suite of over 3000 independent $N$-body simulations that cover a range of cluster mass, metallicity, and half-mass radii. We find stellar-mass black hole tidal disruption events (TDEs) occur at an overall rate of up to roughly $200 \mathrm{Gpc}^{-3} \mathrm{yr}^{-1}$ in young stellar clusters in the local universe. These TDEs are expected to have several characteristic features, namely, fast rise times of order a day, peak X-ray luminosities of at least $10^{44} \mathrm{erg} \mathrm{s}^{-1}$, and bright optical luminosities (roughly $10^{41}-10^{44} \mathrm{erg} \mathrm{s}^{-1}$ ) associated with reprocessing by a disk wind. In particular, we show these events share many features in common with the emerging class of Fast Blue Optical Transients.
\end{abstract}

Unified Astronomy Thesaurus concepts: Astrophysical black holes (98); Young star clusters (1833); Tidal disruption (1696); Transient sources (1851); N-body simulations (1083); X-ray transient sources (1852)

\section{Introduction}

The majority of stars are expected to form in clustered environments such as young star clusters (YSCs; e.g., Carpenter 2000; Lada \& Lada 2003). Several examples of YSCs exist in the Milky Way and the Local Group, and they are expected to be particularly abundant in starburst and interacting galaxies (e.g., Portegies Zwart et al. 2010). As dense stellar systems, YSCs undergo intense dynamical evolution governed by two-body relaxation, similar to their globular cluster (GC) cousins. Unlike GCs, which are massive $\left(\sim 10^{5}-10^{6} M_{\odot}\right)$ and old (ages of $10 \mathrm{Gyr}$ or more), YSCs are generally low mass $\left(\leqslant 10^{5} M_{\odot}\right)$ and short lived-many dissolve in the disk of their host galaxy on timescales of $\mathcal{O}(100 \mathrm{Myr})$ (e.g., Kruijssen et al. 2011). However, before they dissolve, the stellar dynamical processes operating in YSCs make them efficient nurseries for many unusual astrophysical objects.

Over the past decade, the topic of stellar-mass black hole (BH) populations in stellar clusters has seen a boom in interest. On the observational side, a growing number of stellar-mass BH candidates have been observed in Milky Way GCs through both radial velocity measurements (Giesers et al. 2018, 2019) and through X-ray/radio observations (Maccarone et al. 2007; Strader et al. 2012; Chomiuk et al. 2013; Miller-Jones et al. 2015; Shishkovsky et al. 2018). On the theoretical side, stateof-the-art $\mathrm{N}$-body modeling has shown that stellar-mass $\mathrm{BHs}$ form and are retained in the stellar clusters of all masses (e.g., Morscher et al. 2015; Wang et al. 2015; Rodriguez et al. 2016; Banerjee 2017; Arca Sedda et al. 2018; Askar et al. 2018; Weatherford et al. 2020; Kremer et al. 2020b). Furthermore, cluster simulations have revealed that these BHs play a crucial role in the long-term dynamics, core evolution, and survival of

\footnotetext{
${ }^{5}$ NSF Astronomy \& Astrophysics Postdoctoral Fellow.
}

stellar clusters (e.g., Mackey et al. 2007; Breen \& Heggie 2013; Chatterjee et al. 2017; Kremer et al. 2018b, 2019a, 2020b; Ye et al. 2019; Giersz et al. 2019; Wang 2020).

One of the most exciting developments in this field lies in gravitational wave (GW) astrophysics. After formation, BHs are expected to rapidly sink to the center of their host cluster through dynamical friction (e.g., Kulkarni et al. 1993; Sigurdsson 1993; Morscher et al. 2015). Within their host cluster's dense core, $\mathrm{BH}-\mathrm{BH}$ binaries form and subsequently harden through three-body dynamical encounters. Ultimately (depending on the host cluster's escape velocity), these binary $\mathrm{BHs}$ (BBHs) are either dynamically ejected from their host cluster through gravitational recoil or merge inside their host cluster through GW inspiral. Recent studies have shown that YSCs (e.g., Ziosi et al. 2014; Di Carlo et al. 2019a; Banerjee 2021) and old GCs (e.g., Rodriguez et al. 2016; Rodriguez \& Loeb 2018; Antonini \& Gieles 2020; Kremer et al. 2020b) may contribute comparably to the overall BBH merger rate.

Additionally, stellar-mass BHs are expected to dynamically interact with luminous stars in stellar clusters. BH-star encounters are expected to play a crucial role in the formation of both accreting and detached BH binaries (e.g., Ivanova et al. 2010, 2017; Giesler et al. 2018; Kremer et al. 2018a) with properties similar to the $\mathrm{BH}$ candidates detected to date in Milky Way GCs (e.g., Kremer et al. 2019a). Additionally, such dynamical encounters may occasionally cause a star to cross a $\mathrm{BH}$ within its tidal disruption radius, leading to a tidal disruption of the star (Perets et al. 2016; Lopez et al. 2020; Kremer et al. 2019b, 2019c; Samsing et al. 2019; Fragione et al. 2020). These stellar-mass BH tidal disruption events (TDEs) may occur during close encounters of pairs of single stars (i.e., single-single interactions) and also during small- $N$ 
(typically three- or four-body) resonant encounters that occur through binary-mediated dynamical interactions (e.g., Fregeau \& Rasio 2007).

Regardless of the dynamical pathway, these TDEs are expected to lead to transients with fast rise times of roughly a day driven by viscous accretion onto the BH (e.g., Perets et al. 2016). Depending on the assumed accretion efficiency of the subsequently formed accretion disk, outflows associated with disk wind mass loss are expected to reprocess the inner-disk radiation on a timescale of a few to 10 days, leading to peak bolometric luminosities up to roughly $10^{44} \mathrm{erg} \mathrm{s}^{-1}$ that peak in the optical (Kremer et al. 2019c). In the case of extremely efficient energy release in the form of a jet, a bright X-ray or $\gamma$ ray flare may result with the overall phenomenology possibly resembling ultra-long gamma-ray bursts (Perets et al. 2016). These TDEs are expected to occur in GCs at rates of roughly 3-10 $\mathrm{Gpc}^{-3} \mathrm{yr}^{-1}$ (Perets et al. 2016; Lopez et al. 2020; Kremer et al. 2019c) and at similar rates in both nuclear star clusters (Fragione et al. 2020) and in stellar triples under the influence of Lidov-Kozai oscillations (Fragione et al. 2019).

In this paper, we examine the tidal disruption of mainsequence stars by stellar-mass BHs in YSCs, in particular investigating the low-mass cluster regime, which is expected to dominate (by total number of clusters) the overall cluster mass function. This expands upon earlier work on the topic that was limited to old and massive GCs. We compile an extensive suite of $N$-body cluster models for various cluster masses and explore both TDE rates as well as characteristic properties.

Recent, current, and upcoming high-cadence surveys such as the Palomar Transient Factory (e.g., Law et al. 2009), the Zwicky Transient Facility (e.g., Bellm et al. 2019), All-Sky Automated Survey for Supernovae (e.g., Shappee et al. 2014), Asteroid Terrestrial-impact Last Alert System (e.g., Tonry et al. 2018), Panoramic Survey Telescope and Rapid Response System (e.g., Chambers et al. 2016), and the Vera Rubin Observatory (e.g., LSST Science Collaboration et al. 2009) are ushering in an unprecedented era in transient astronomy. Thus, the catalog of observed transients of both known and unknown origin is growing and will continue to grow rapidly. We conclude this study by examining the electromagnetic (EM) features of stellar-mass BH TDEs and compare these events specifically with the emerging class of Fast Blue Optical Transients (FBOTs; e.g., Drout et al. 2014; Arcavi et al. 2016; Pursiainen et al. 2018; Rest et al. 2018; Margutti et al. 2019; Coppejans et al. 2020; Ho et al. 2020). On the basis of event rates, host-galaxy properties, and overall transient features such as rise times and peak luminosities, we demonstrate that stellarmass BH TDEs may indeed be a viable mechanism for FBOTlike events.

In Section 2, we describe the methods we use to model stellar clusters. In Sections 3.1 and 3.2, we estimate TDE rates occurring through single-single and binary-mediated encounters, respectively, and compare the rates estimated from our $\mathrm{N}$ body models with simple analytic estimates. In Section 3.3, we compute the overall TDE rates at various cosmological distances and in Section 3.4, we describe how TDE properties may vary with cluster metallicity. In Section 4, we discuss of the expected outcome of stellar-mass BH TDEs, specifically describing the basic properties of disk formation and evolution and the associated EM signatures. We also compare these features with observed properties of FBOTs. We discuss our results and conclude in Section 5.

\section{2. $N$-body Models of Young Clusters}

To model the evolution of YSCs, we use the Hénon-type Monte Carlo code CMC (Joshi et al. 2000; Pattabiraman et al. 2013; Kremer et al. 2020b). CMC includes various physical processes necessary to study both large scale cluster dynamics and the formation and evolution of stellar-mass BHs, including two-body relaxation, stellar, and binary star evolution (computed using updated versions of SSE and BSE; Hurley et al. $2000,2002)$, and direct integration of small- $N$ resonate encounters (Fregeau \& Rasio 2007) including post-Newtonian effects (Rodriguez et al. 2018).

To compute compact object (BH and neutron star; NS) masses, we adopt the stellar wind prescriptions of Vink et al. (2001) to determine the final stellar mass at the moment of core collapse and adopt the "rapid" supernova explosion models (Fryer et al. 2012) to compute NS and BH masses modified to include the prescriptions for (pulsational) pair-instability supernovae described in Belczynski et al. (2016). BH and NS natal kicks are computed as in Kremer et al. (2020b).

In order to treat stellar-mass BH TDEs, we adopt the same prescriptions as in Kremer et al. (2019c). In short, if a dynamical encounter involving at least one $\mathrm{BH}$ and one star ${ }^{6}$ leads to a BH-star pericenter passage, $r_{p}$, within the star's tidal disruption radius

$$
r_{\mathrm{TD}}=\left(\frac{m_{\mathrm{BH}}}{m_{\star}}\right)^{1 / 3} R_{\star},
$$

where $m_{\mathrm{BH}}$ is the $\mathrm{BH}$ mass, and $m_{\star}$ and $R_{\star}$ are the stellar mass and radius, respectively, we assume a TDE occurs. ${ }^{7}$ At this point, we record the stellar properties and then assume the star is instantaneously destroyed. In reality, especially if the TDE occurs during a multi-body resonant encounter, TDEs may affect the hydrodynamic evolution of their dynamical encounters. These more complex effects are well beyond the computational scope of an $\mathrm{N}$-body code like CMC, but see e.g., Lopez et al. (2020) for a discussion.

We apply this TDE prescription only for BH-star interactions. For close encounters of star-star pairs, we allow the stars to interact only in the direct collision limit: we assume a sticky sphere collision (i.e., zero mass loss) occurs if $r_{p}<R_{1}+R_{2}$, where $R_{1}$ and $R_{2}$ are the stellar radii. See Kremer et al. (2020a) for further details of our treatment of star-star collisions. We record TDEs/collisions that occur during both single-single encounters and binary-mediated dynamical encounters that are integrated directly using Fewbody. For further details, see Fregeau \& Rasio (2007) and Kremer et al. (2019c, 2020a).

In all models, we assume a static Milky Way-like external tidal field representative of the solar neighborhood (i.e., located at a distance of $8 \mathrm{kpc}$ from the Galactic center). In reality, this choice likely underestimates the role of external tides on the long-term cluster evolution as it does not incorporate the effects of massive perturbers (e.g., molecular clouds), which may

\footnotetext{
6 Here, we are specifically interested in disruption of main-sequence stars and do not consider the disruption of giants that occur roughly a factor of 10 less frequently (e.g., Kremer et al. 2019c). Henceforth, we use the term "star" to mean a main-sequence star. For a discussion of the interaction of BHs with giants, see Ivanova et al. (2010, 2017) and Kremer et al. (2019c).

In reality, the tidal disruption radius of a particular object likely depends also upon the object's stellar structure. In particular, this dependence may change as stars evolve and develop a more pronounced core-envelope structure. We reserve inclusion of these more detailed effects for future study and note that these effects are unlikely to affect the results presented here significantly.
} 
accelerate the cluster disruption (e.g., Gieles et al. 2006). We do not model here the dynamics of the final phase of cluster dissolution. As in Di Carlo et al. (2019a), we integrate our clusters to a maximum age of 150-500 Myr, depending on the cluster mass (allowing more massive clusters to evolve longer to reflect their long relaxation times; e.g., Heggie \& Hut 2003). Indeed, assuming a maximum age of $500 \mathrm{Myr}$ is appropriate as our focus here lies primarily on young clusters as opposed to long-lived globular clusters with ages of $10 \mathrm{Gyr}$ or more (see Portegies Zwart et al. 2010, for further discussion). Furthermore, real star clusters are formed in a complicated interaction between gas and gravity (e.g., Bate et al. 2003), which, in general, is poorly understood. In $\mathrm{CMC}$, we neglect the initial gas-rich phase of cluster evolution and instead assume a single starburst creates all stars. In particular, we do not consider expulsion of primordial gas that occurs on a dynamical timescale at early times and the possible consequences on the cluster dynamics/survival (e.g., the "infant mortality" effect; Lada \& Lada 2003).

We consider initial cluster masses in the range of $6000-4.8 \times 10^{5} M_{\odot}$, reflective of the YSC masses observed in local universe (e.g., Lada \& Lada 2003; Portegies Zwart et al. 2010). For all models, we adopt a standard Kroupa (2001) initial mass function with a mass range of $0.08-150 M_{\odot}$. We assume all models are initially fit to a King model with concentration parameter $W_{0}=5$ (King 1962).

We adopt two values for the cluster initial virial radius, $r_{v}$. In the first limit, we assume a constant $r_{v}=1 \mathrm{pc}$ for all cluster masses (e.g., Portegies Zwart et al. 2010). In the second limit, we follow the phenomenological results of Marks \& Kroupa (2012) that showed an initial cluster half-mass radii, $r_{\mathrm{h}}$ (a reasonable proxy for $r_{v}$ ), exhibit a weak dependence on total cluster mass:

$$
r_{\mathrm{h}}=0.1_{-0.04}^{+0.07} \mathrm{pc}\left(\frac{M_{\mathrm{cl}}}{M_{\odot}}\right)^{0.13 \pm 0.04} .
$$

As the virial radii given by Equation (2) are a factor of roughly 2 smaller than the $r_{v}=1 \mathrm{pc}$ assumption, the models adopting this relation are roughly an order of magnitude denser than their $r_{v}=1 \mathrm{pc}$ counterparts. In this case, the various dynamical processes, including stellar-mass BH TDEs occur at an increased rate under the Marks \& Kroupa (2012) assumption, as will be discussed further in Section 3.

For simplicity, we assume zero primordial stellar binaries in all models in this study. Under this assumptions, all TDEs occur as a result of well-understood dynamical processes, with no assumptions required regarding the uncertain properties of primordial stellar binaries in clusters. However, we note that primordial binaries will likely lead to an increase in TDE rate (for example, see Fregeau \& Rasio (2007), which explored the role of primordial binaries in the similar topic of stellar collisions). In this case, the results of this study may be viewed as a conservative lower limit on the true TDE rate in YSCs. We return to this question in Section 5.

Finally, to increase the robustness of our results, we run a large number of independent realizations of each set of cluster initial conditions. In total, we produce 3010 independent models. The complete list of models, including initial conditions and number of TDEs, is shown in Table 1 .

\section{Results}

In this section, we show the results of our suite of $N$-body models. In Sections 3.1 and 3.2, we discuss TDEs that occur during single-single and binary-single dynamical encounters, respectively, and compare them to simple analytic estimates. In Section 3.3, we examine the properties of TDEs and how these properties vary with both the cluster metallicity and initial density. Finally, in Section 3.4, we estimate the overall rates of TDEs at various redshifts.

\subsection{Single-Single TDEs}

We first discuss the case of TDEs occurring during singlesingle encounters between a $\mathrm{BH}$ and an MS star. For a given cluster with $N$ total stars and half-mass radius $r_{\mathrm{h}}$, the rate of TDEs occurring through single-single dynamical encounters can be estimated as

$$
\Gamma_{\mathrm{ss}} \approx n_{\star} \Sigma_{\mathrm{ss}} \sigma_{v} N_{\mathrm{BH}},
$$

where $N_{\mathrm{BH}}$ is the total number of BHs in the cluster (which, in general, are all found within the half-mass radius due to mass segregation; e.g., Morscher et al. 2015), $n_{\star} \approx N / r_{\mathrm{h}}^{3}$ is the number density, and $\sigma_{v}$ is the cluster's velocity dispersion. $\Sigma_{\mathrm{ss}}$ is the cross section for a single-single TDE, given by

$$
\Sigma_{\mathrm{ss}}=\pi R_{\mathrm{TD}}^{2}\left[1+\frac{2 G\left(m_{\mathrm{BH}}+m_{\star}\right)}{R_{\mathrm{TD}} \sigma_{v}^{2}}\right],
$$

where $m_{\mathrm{BH}}$ and $m_{\star}$ are the typical $\mathrm{BH}$ and stellar masses and $R_{\mathrm{TD}}$ is the tidal disruption radius given by Equation (1). For a cluster with a Kroupa (2001) IMF, we can take $m_{\star} \approx 0.6 M_{\odot}$. For high-metallicity clusters $\left(Z \approx Z_{\odot}\right)$, we can take $m_{\mathrm{BH}} \approx 10 M_{\odot}$, while for low-metallicity clusters $\left(Z \lesssim 0.1 Z_{\odot}\right)$, $m_{\mathrm{BH}} \approx 25 M_{\odot}$ is more appropriate (e.g., Kremer et al. 2020b).

Assuming the cluster is initially in virial equilibrium ( $\sigma_{v} \approx \sqrt{G M_{\mathrm{cl}} / r_{\mathrm{h}}}$, where $M_{\mathrm{cl}} \approx m_{\star} N$ is the total cluster mass), assuming all encounters occur in the gravitational focusing regime (the second term in the brackets of Equation (4) dominates), and taking $m_{\mathrm{BH}} \gg m_{\star}$, we can rewrite Equation (3) as

$$
\begin{aligned}
\Gamma_{\mathrm{ss}} \approx & 0.2 \mathrm{Gyr}^{-1}\left(\frac{M_{\mathrm{cl}}}{10^{4} M_{\odot}}\right)^{1 / 2}\left(\frac{r_{\mathrm{h}}}{1 \mathrm{pc}}\right)^{-5 / 2} \\
& \times\left(\frac{m_{\star}}{0.6 M_{\odot}}\right)^{-4 / 3}\left(\frac{m_{\mathrm{BH}}}{10 M_{\odot}}\right)^{4 / 3}\left(\frac{R_{\star}}{0.6 R_{\odot}}\right)\left(\frac{N_{\mathrm{BH}}}{10}\right) .
\end{aligned}
$$

For a Kroupa (2001) IMF, we expect roughly $10^{-3} \mathrm{BHs}$ to form per star, giving us $N_{\mathrm{BH}} \approx 10^{-3} N$. In this case, we obtain the analytic scaling

$$
\Gamma_{\mathrm{ss}} \approx 0.2 \mathrm{Gyr}^{-1}\left(\frac{M_{\mathrm{cl}}}{10^{4} M_{\odot}}\right)^{3 / 2} .
$$

In Figure 1, we show as open circles the rates of such encounters as a function of cluster mass as determined from our $\mathrm{N}$-body models. To isolate specifically the effect of cluster mass, we utilize only the first eight sets of simulations listed in Table 1 (models $a-h$ ), which have fixed metallicity $\left(Z_{\odot}\right)$ and virial radius $(1 \mathrm{pc})$. To calculate the model rates, we simply count the total number of single-single TDEs in all models of a given cluster mass, then divide by the total number of models 
Table 1

List of $N$-body Models

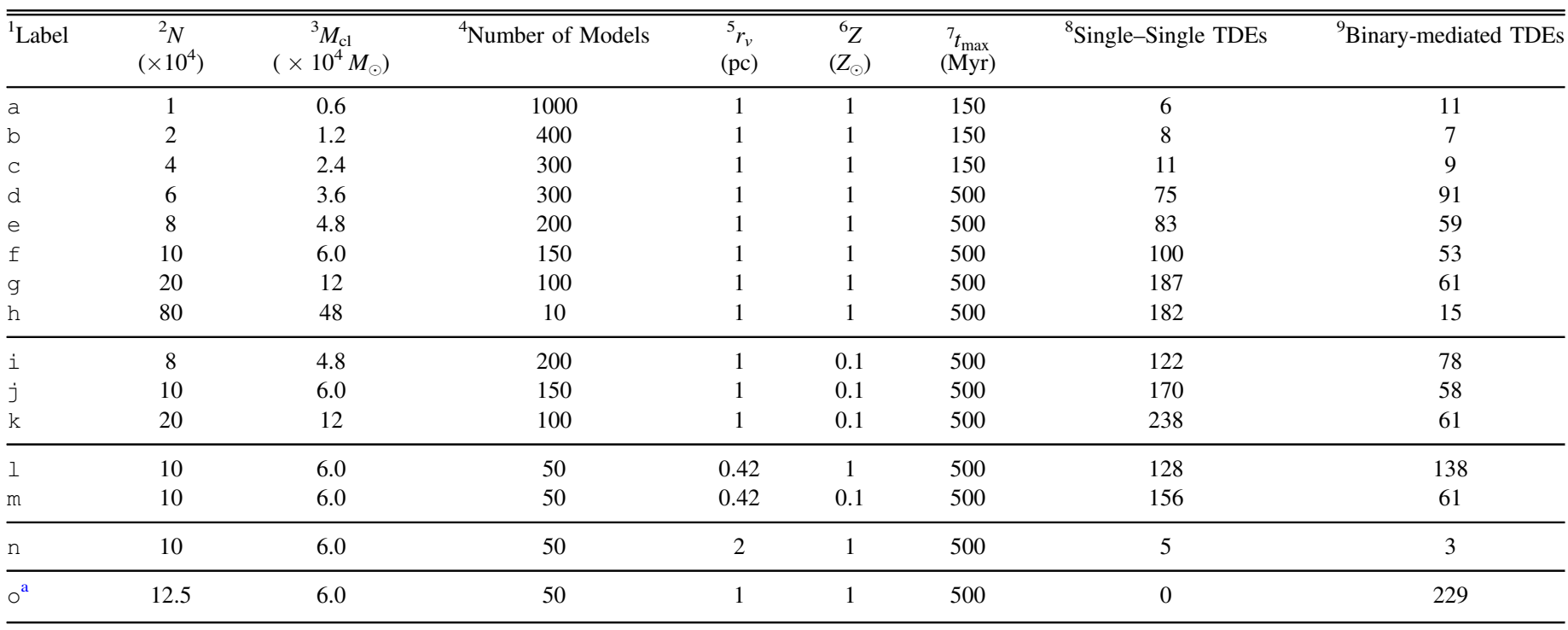

Notes. All models computed in this study. In Columns 2 and 3, we list the initial number of stars and cluster mass, respectively. In Column 4, we list the total number of independent realizations computed for the given set of initial conditions. In Columns 5, 6, and 7, we list the initial virial radius, metallicity, and maximum integration time, respectively. In Columns 8 and 9, we list the total number of TDEs occurring through single-single and binary-single encounters, respectively.

${ }^{a}$ Unlike models $a-n$, which adopt zero primordial binaries, model o adopts a 100\% primordial binary fraction. We discuss this further in Section 5.

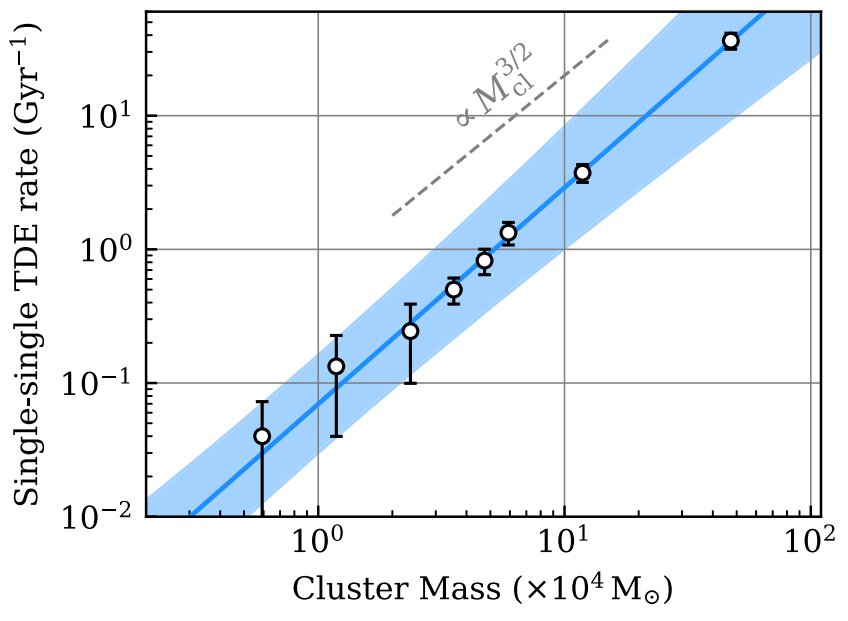

Figure 1. TDE rate per cluster as a function of initial cluster mass using models $\mathrm{a}-\mathrm{h}$ shown in Table 1 (assuming $r_{h}=1 \mathrm{pc}$ ). Open circles denote rates computed from the suite of $N$-body models and the solid blue curve shows the best-fit relation of Equation (7). The blue shaded region denotes the $90 \%$ confidence interval from the least squares fit. The gray dashed line shows the $\propto M_{\mathrm{cl}}^{3 / 2}$ analytic scaling from Equation (6).

and by the total integration time for the given cluster mass (see Table 1). Error bars denote $2 \sigma$ from the mean, assuming a Poisson distribution.

From a least squares fit, we find these data are best fit by the power-law relation

$$
\Gamma_{\mathrm{ss}}^{\text {model }}=0.07 \pm 0.01 \mathrm{Gyr}^{-1}\left(\frac{M_{\mathrm{cl}}}{10^{4} M_{\odot}}\right)^{1.6 \pm 0.16} .
$$

This fit is shown as the blue curve in Figure 1, with the blue bands denoting the $90 \%$ confidence interval from the least squares fit. For comparison, we show as the gray dashed line in
Figure 1 the $\Gamma \propto M_{\mathrm{cl}}^{3 / 2}$ scaling relation derived from the simple analytic estimate in Equation (6).

In Equation (6), we have assumed a constant $r_{\mathrm{h}} \approx 1 \mathrm{pc}$ is typical for all YSCs (see, e.g., Portegies Zwart et al. 2010). Alternatively, as discussed in Section 2, $r_{\mathrm{h}}<1 \mathrm{pc}$ may be more appropriate and furthermore, $r_{\mathrm{h}}$ may exhibit a weak dependence on total cluster mass. To explore the possibility, we ran an additional set of models (group 1 in Table 1) with initial $r_{v}=0.42 \mathrm{pc}$, reflective of the $r_{\mathrm{h}}-M_{\mathrm{cl}}$ relation of Marks \& Kroupa (2012). Given the phenomenological relation from Marks \& Kroupa (2012) predicts cluster radii a factor of roughly 2 lower than the $r_{\mathrm{h}}=1 \mathrm{pc}$ assumption, the following rate, $\Gamma_{\mathrm{ss}}^{\text {phenom }}$, is higher than that estimated from Equation (7).

Combining the phenomenological $r_{\mathrm{h}}-M_{\mathrm{cl}}$ relation of Marks \& Kroupa (2012) (Equation (2)) with Equations (3) and (4), we expect $\Gamma_{\mathrm{ss}}^{\text {phenom }} \propto M_{\mathrm{cl}}^{1.175}$. Scaling to the rate identified from the models in group 1 , we can then write

$$
\Gamma_{\mathrm{ss}}^{\text {phenom }} \approx 0.63 \mathrm{Gyr}^{-1}\left(\frac{M_{\mathrm{cl}}}{10^{4} M_{\odot}}\right)^{1.175} .
$$

\subsection{Binary-mediated TDEs}

In addition to TDEs occurring through single-single encounters, TDEs may also take place during binary-mediated resonant encounters involving at least one $\mathrm{BH}$ and one star. As discussed in Section 2, we do not include stellar binaries in this study. The only binaries formed are BH binaries assembled through three-body encounters (for simplicity, three-body binary formation is allowed only for BHs in our models; e.g., Morscher et al. 2015). In this case, the binary-mediated TDEs discussed in this subsection are those that occur specifically during binary-single resonant encounters between a BBH and a single MS star.

Using a similar calculation to that performed for the singlesingle rate estimate, the rate of TDEs during BBH-star binary- 


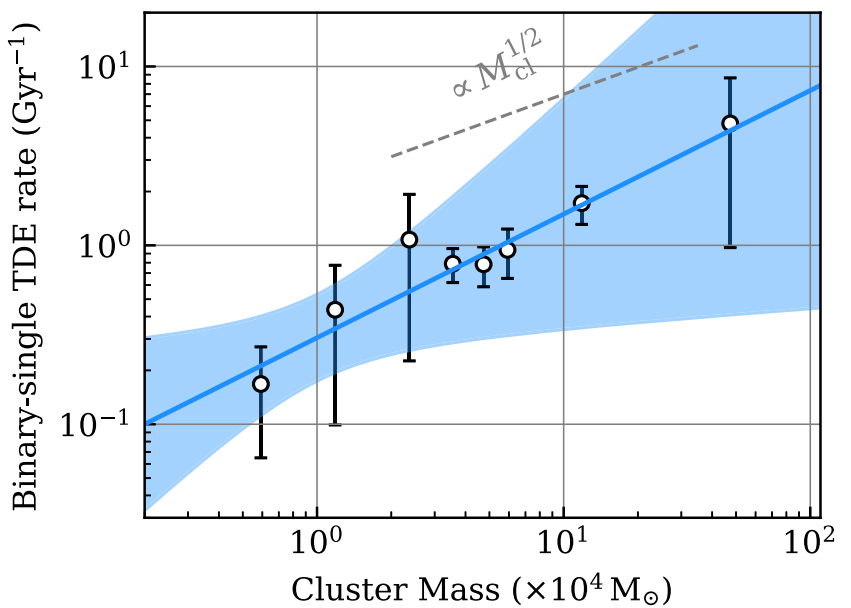

Figure 2. Same as Figure 1 but for TDEs occurring during binary-single encounters. Here, the blue curve shows the best-fit relation of Equation (13) (as in Figure 1, the blue shaded region denotes the $90 \%$ confidence interval from the least squares fit) and the gray dashed line shows the $\propto M_{\mathrm{cl}}^{1 / 2}$ analytic scaling from Equation (11).

single encounters can be written as

$$
\Gamma_{\mathrm{bs}} \approx n_{\star} \Sigma_{\mathrm{bs}} \sigma_{v} N_{\mathrm{BBH}} P_{\mathrm{TD}}
$$

Here, $\Sigma_{\mathrm{bs}}$ is the cross section for binary-single encounters

$$
\Sigma_{\mathrm{bs}}=\pi a_{\mathrm{BBH}}^{2}\left[1+\frac{2 G\left(m_{\mathrm{BBH}}+m_{\star}\right)}{a_{\mathrm{BBH}} \sigma_{v}^{2}}\right],
$$

where $a_{\mathrm{BBH}}$ is the $\mathrm{BBH}$ semimajor axis and $m_{\mathrm{BBH}} \approx 2 m_{\mathrm{BH}}$ is the mass of the BBH. $N_{\mathrm{BBH}}$ is the total number of BBHs in the cluster. As shown in a number of recent analyses (e.g., Morscher et al. 2015; Chatterjee et al. 2017; Banerjee 2018), $N_{\mathrm{BBH}}$ is expected to be roughly independent of the total number of BHs in the cluster as well as the cluster's total mass, such that the total number of dynamically formed $\mathrm{BBHs}$ present at any given time never exceeds a few. Here, we assume $N_{\mathrm{BBH}} \approx 2$.

Finally, $P_{\mathrm{TD}}$ is the probability that a given $\mathrm{BBH}-$ star resonant encounter leads to a TDE. We follow the results of Darbha et al. (2018), which showed that for asymmetric mass ratio binary-single encounters, $P_{\mathrm{TD}}$ is roughly proportional to $R_{\mathrm{TD}} / a_{\mathrm{BBH}}$. Note that this same scaling is found in the equal mass case (e.g., Samsing et al. 2017; Samsing 2018). Here, we take $P_{\mathrm{TD}}=2 R_{\mathrm{TD}} / a_{\mathrm{BBH}}$ as in Samsing et al. (2019). We can then rewrite Equation (9) as

$$
\Gamma_{\mathrm{bs}} \approx 0.3 \mathrm{Gyr}^{-1}\left(\frac{M_{\mathrm{cl}}}{10^{4} M_{\odot}}\right)^{1 / 2},
$$

where, as before, we have assumed $r_{\mathrm{h}} \approx 1 \mathrm{pc}, m_{\mathrm{BH}} \approx 10 M_{\odot}$, $m_{\star} \approx 0.6 M_{\odot}$, and $R_{\star} \approx 0.6 R_{\odot}$, independent of the cluster mass.

Comparing to Equation (6), we find $\Gamma_{\mathrm{bs}} / \Gamma_{\mathrm{ss}} \propto N_{\mathrm{BH}}^{-1} \propto M_{\mathrm{cl}}^{-1}$. Thus, lower-mass clusters with fewer BHs feature a higher $\mathrm{BBH}$ TDE rate per $\mathrm{BH}$ (and therefore also per star) compared to higher-mass clusters with more BHs. Thus, given that lowermass clusters also dominate by number the overall cluster mass function, they are an ideal environment to find BBH TDEs. We return to the question of overall and relative rates in Section 3.3.
In Figure 2, we show the rates of these events as a function of cluster mass as determined from our $N$-body models. As in Figure 1, the rate is computed as the total number of binarysingle TDEs in all models of a given cluster mass, divided by the total number of models and by the time duration, $\Delta t$. Unlike in the single-single case, where the necessary dynamical encounters begin immediately, in the binary-single case we must first wait for BHs to mass segregate to the center so that the target $\mathrm{BBHs}$ can form through three-body encounters. For each model, we define this timescale, $t_{3 \mathrm{bb}}$, simply as the moment the first BBH forms in that model. Then, $\Delta t=t_{\max }-t_{3 \mathrm{bb}}$. Typically, $t_{3 \mathrm{bb}}$ is of order $100 \mathrm{Myr}$ (see also Sigurdsson 1993; Morscher et al. 2015).

Again performing a least squares fit, we find these data are best fit by the power-law relation

$$
\Gamma_{\mathrm{bs}}^{\text {model }}=0.69 \pm 0.36 \mathrm{Gyr}^{-1}\left(\frac{M_{\mathrm{cl}}}{10^{4} M_{\odot}}\right)^{0.3 \pm 0.09}
$$

which can be compared with the simple analytic estimate of Equation (11). As with the single-single case, we find the analytic estimate recovers reasonably well the rate inferred from the $N$-body modeling.

As before, we can also estimate a phenomenological rate, $\Gamma_{\mathrm{bs}}^{\text {phenom }}$, assuming the relation of Marks \& Kroupa (2012). Combining Equations (2) and (11), we expect $\Gamma_{\mathrm{bs}}^{\mathrm{phenom}} \propto M_{\mathrm{cl}}^{0.175}$. Again normalizing to the binary-single TDE rate estimated in the models from group 1 in Table 1 , we can write

$$
\Gamma_{\mathrm{bs}}^{\mathrm{phenom}} \approx 4.4 \mathrm{Gyr}^{-1}\left(\frac{M_{\mathrm{cl}}}{10^{4} M_{\odot}}\right)^{0.125} .
$$

Because we assume that $\mathrm{BH}$ binaries form exclusively through three-body encounters, Equations (12) and (13) are relevant only for those clusters with at least three BHs at birth, so that at least one BBH can form. Again, assuming roughly 1 BH forms per 1000 stars (Kroupa 2001), this requires $N \gtrsim 3000$ or $M_{\mathrm{cl}} \gtrsim 2000 M_{\odot}$. For clusters with $M_{\mathrm{cl}} \lesssim 2000 M_{\odot}$, Equations (12) and (13) are no longer applicable and the binary-single TDE rate is zero. We return to this point in Section 3.3 when we compute the total TDE rate by integrating over the full cluster mass function.

\subsubsection{BBH Orbital Separations}

Given that the binary-single TDE rate is expected to be roughly independent of the binary orbital separation, the distribution of $a_{\mathrm{BBH}}$ for BBHs that undergo TDEs is expected to follow the semimajor axis for all BBHs in a cluster of a given mass (e.g., Kremer et al. 2019b; Samsing et al. 2019). In Figure 3, we show the distribution of $a_{\mathrm{BBH}}$ for all BBHs found in models $\mathrm{a}^{-\mathrm{h}}$ (various initial masses, but fixed $r_{v}$ and $Z$ ). These distributions are determined by two primary physical processes:

Three-body formation: The maximum semimajor axis of a binary formed through a three-body encounter is determined by the hard-soft boundary $a_{\mathrm{HS}} \propto m_{\mathrm{BH}} / \sigma_{v}^{2}$ (e.g., Morscher et al. 2015). Assuming $\sigma_{v}^{2} \propto M_{\mathrm{cl}}$, we expect, in general, more massive clusters will produce more compact BBHs compared to lower-mass clusters (assuming a fixed cluster virial radius).

Ejection from dynamical recoil: Once a $\mathrm{BBH}$ is formed, it will (on average) harden through subsequent binary-mediated 


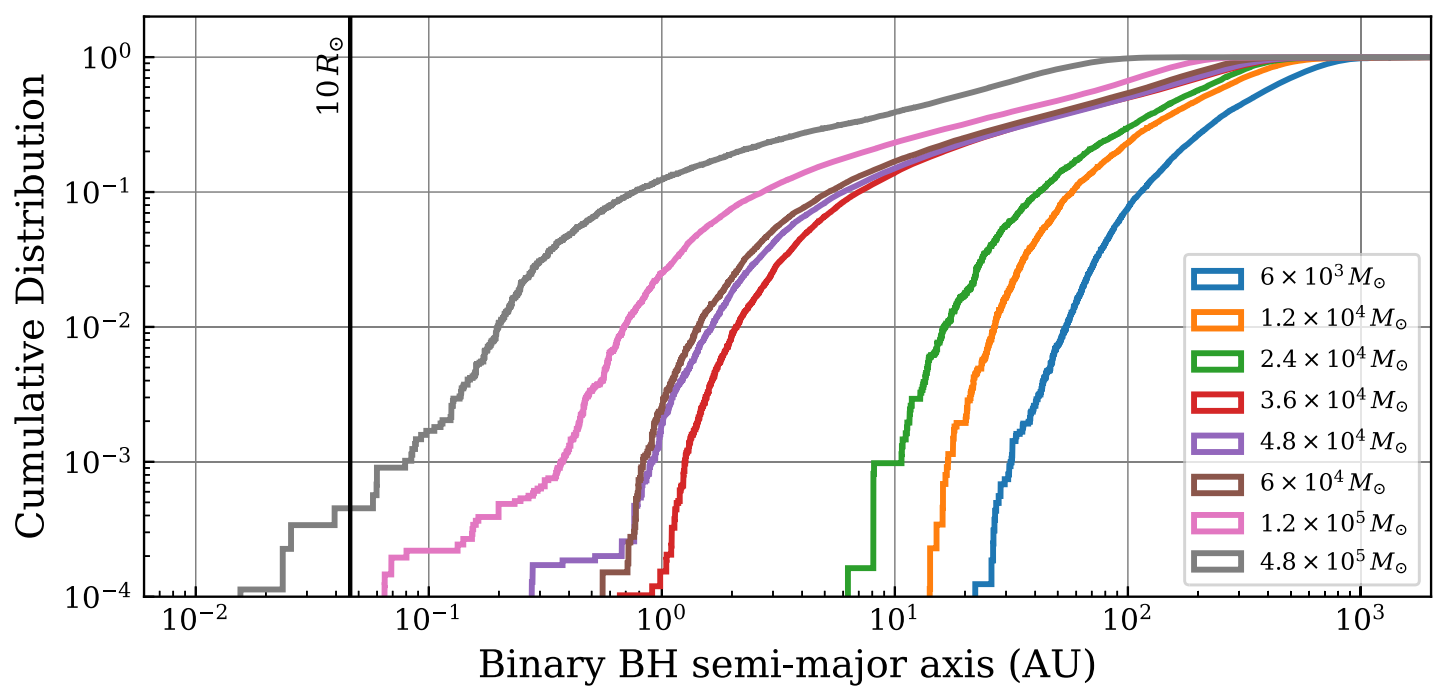

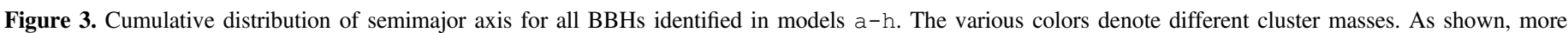

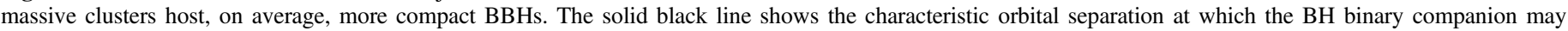
interrupt the TDE light curve, as described in the text.

encounters with other BHs and stars in the cluster core (e.g., Sigurdsson 1993; Morscher et al. 2015; Rodriguez et al. 2016). Following a dynamical encounter, the BBH will receive a dynamical recoil kick with a magnitude comparable to the $\mathrm{BBH}$ orbital velocity, $v_{\text {recoil }}^{2} \propto a_{\mathrm{BBH}}^{-1}$. Thus, as a BBH hardens, it attains increasingly large dynamical recoil kicks. Eventually, $v_{\text {recoil }}$ is sufficiently large for the binary to be ejected from the cluster. This is set by the cluster's escape velocity, $v_{\text {esc }}^{2} \propto M_{\mathrm{cl}} / r_{\mathrm{h}}$. As a result, in lower-mass clusters, BBHs will be dynamically ejected before they can harden as far as is possible in higher-mass clusters.

As a consequence of these two processes, we expect the BBH semimajor axis distribution to shift toward lower values in increasingly massive clusters. Indeed, this is shown in Figure 3.

One exciting possibility proposed in Lopez et al. (2020), Samsing et al. (2019), and Kremer et al. (2019b) is that these binary-mediated TDEs may be used to indirectly probe properties of the underlying BBH population, if the corresponding EM signal can be detected. The basic idea is the second $\mathrm{BH}$ produces breaks in the light curve on timescale comparable to the $\mathrm{BBH}$ orbital period. This idea has been illustrated in the supermassive $\mathrm{BH}(\mathrm{SMBH})$ regime using numerical techniques (e.g., Liu et al. 2009; Coughlin et al. 2017), and one SMBH candidate has proposed (TDE J1201+30), from which the authors were able to constrain the SMBH binary orbital period (Liu et al. 2014). This process likely requires $a_{\mathrm{BBH}}$ to be comparable to the disk radius, which in turn will be comparable to the tidal disruption radius. For reference, we show $r_{\mathrm{TD}} \approx 10 R_{\odot}$ as a solid black line in Figure 3 (see Equation (1)). As shown in the figure, this process is likely only possible in the most massive clusters explored here $\left(M_{\mathrm{cl}} \gtrsim 10^{5} M_{\odot}\right)$. Even for our massive cluster simulations, we find that only $\approx 0.1 \%$ of all BBHs meet this criterion. Thus, we conclude that the presence of a second $\mathrm{BH}$ is unlikely to significantly affect the TDE dynamics and subsequent lightcurve evolution.

Although it appears this possibility is not relevant in typical YSCs, we can speculate that more massive clusters such as nuclear star clusters with masses of $10^{7} M_{\odot}$ or larger may be ideal environments for this processes, given that more massive clusters should be able to host even more compact BBHs. We reserve a more detailed study of this possibility for future study, and direct the reader to Fragione et al. (2020) for a discussion of TDEs in the nuclear star cluster regime.

\subsection{Estimating the Total Event Rate}

The functional form for the initial mass function of YSCs is expected to be well represented by a power-law distribution (e.g., Lada \& Lada 2003) with a possible exponential truncation above cluster masses of roughly $M_{\text {cut }} \approx 10^{6} M_{\odot}$ (e.g., Portegies Zwart et al. 2010):

$$
\frac{d N}{d M_{\mathrm{cl}}} \propto M_{\mathrm{cl}}^{-2} \exp \left(-M_{\mathrm{cl}} / M_{\mathrm{cut}}\right)
$$

As we are interested here primarily in the low-mass tail of the mass function $\left(\lesssim 10^{5} M_{\odot}\right)$, the specific value of $M_{\text {cut }}$ is not relevant to this study.

We can then compute the total TDE rate from a realistic population of YSCs in Milky Way-like galaxies by integrating the rate per cluster (Equation (5)) over the cluster mass function:

$$
\Gamma_{\mathrm{tot}}=\int_{M_{\mathrm{low}}}^{M_{\mathrm{high}}} \frac{\Gamma_{\mathrm{cl}}}{M_{\mathrm{cl}}} \frac{d N}{d M_{\mathrm{cl}}} \Delta t \rho_{\mathrm{SF}} f_{\mathrm{SF}} d M_{\mathrm{cl}}
$$

The integration limits represent the assumed range in cluster masses; we assume $M_{\text {low }}=100 M_{\odot}$ and $M_{\text {high }}=10^{5} M_{\odot}$ (Lada \& Lada 2003). By definition, in order for TDEs to occur through the binary-single channel discussed in Section 3.2, BBHs must have formed in the cluster. As discussed in Section 3.2, because we assume that BBHs form exclusively through three-body encounters, this requires at least three BHs be present in the cluster at birth, which in turn requires $M_{\mathrm{cl}} \gtrsim 2000 M_{\odot}$. Thus, to compute the rate of TDEs occurring through binary-single encounters, we use $M_{\text {low }}=2000 M_{\odot}$ (keeping the overall normalization of Equation (14) the same as before). Note that this mass requirement automatically takes 
Table 2

Volumetric TDE Rates

\begin{tabular}{lccc}
\hline \hline$r_{\mathrm{h}}$ Prescription & $z=0$ & $\begin{array}{c}z=1 \\
\left(\mathrm{Gpc}^{-3} \mathrm{yr}^{-1}\right)\end{array}$ & $z=2.5$ \\
\hline \multicolumn{4}{c}{ Single-single TDE rate: } \\
\hline$r_{\mathrm{h}}=1 \mathrm{pc}$ & 1.1 & 7.1 & 12.1 \\
Marks \& Kroupa (2012) & 27.6 & 184.3 & 313.3 \\
\hline \multicolumn{4}{c}{ Binary-single TDE rate: } \\
\hline$r_{\mathrm{h}}=1 \mathrm{pc}$ & 19.3 & 218.3 \\
Marks \& Kroupa (2012) & 157.7 & 128.4 & 1787.0 \\
\hline
\end{tabular}

Note. Volumetric event rates of TDEs occurring through both single-single and binary-single encounters in YSCs in the local universe $(z=0)$, at $z=1$, and at peak star formation $(z \approx 2.5)$. We show both rates calculated assuming constant cluster half-light radii, $r_{\mathrm{h}}=1 \mathrm{pc}$ and assuming the $r_{\mathrm{h}}-M_{\mathrm{cl}}$ relation of Marks \& Kroupa (2012) (Equation (2)).

care of the additional requirement that the cluster not dissolve before the first BBHs begin to form.

$\Delta t$ is the cluster disruption timescale, $t_{\mathrm{dis}}$, which depends upon the location of the cluster in its host galaxy's tidal field, as well as on more complex phenomena such as, e.g., tidal shocks and interactions with giant molecular clouds as mentioned in Section 2. Here, we adopt the following relation from Lamers et al. (2005):

$$
t_{\mathrm{dis}} \simeq 810\left(\frac{M_{\mathrm{cl}}}{10^{4} M_{\odot}}\right)^{0.62}\left(\frac{\rho_{\mathrm{amb}}}{M_{\odot} \mathrm{pc}^{-3}}\right)^{-0.5} \mathrm{Myr},
$$

where $\rho_{\mathrm{amb}} \approx M_{\odot} \mathrm{pc}^{-3}$ is the assumed local ambient density.

Again, as we are specifically interested in young clusters with ages less than roughly $500 \mathrm{Myr}$, we take

$$
\Delta t=\min \left(t_{\mathrm{dis}}, 500 \mathrm{Myr}\right) \text {. }
$$

For TDEs occurring during binary-single encounters, we must also incorporate the timescale for $\mathrm{BBH}$ formation to begin. In Section 3.2, we computed this timescale directly from the models. Here, for simplicity (and as motivated by the results from the models), we assume $\mathrm{BBH}$ formation occurs after roughly $100 \mathrm{Myr}$ for all cluster masses (see also, e.g., Sigurdsson 1993). In this case, for binary-single TDEs, $\Delta t=\min \left(t_{\mathrm{dis}}, 500 \mathrm{Myr}\right)-100 \mathrm{Myr}$.

$\rho_{\mathrm{SF}}$ is the assumed cosmological density of the star formation rate (SFR). We adopt the SFR of Hopkins \& Beacom (2006). Specifically, this study finds $\rho_{\mathrm{SF}} /\left[M_{\odot} \mathrm{yr}^{-1} \mathrm{Mpc}^{-3}\right]=1.5 \times 10^{-2}, 0.1$, and 0.2 at redshift $z=0,1$, and 2.5 (peak star formation), respectively. $f_{\mathrm{SF}}$ is the fraction of the SFR assumed to occur in star clusters. For lowmass clusters $\left(M_{\mathrm{cl}}<10^{5} \odot\right)$ we assume $f_{\mathrm{SF}}=0.8$ (Lada \& Lada 2003).

Finally, $\Gamma$ is the TDE rate per cluster of a given mass, $M_{\mathrm{cl}}$. For this we adopt the scaling relations derived in Section 3 (Equations (7) and (12)), for the single-single and binarysingle cases, respectively, assuming constant $r_{\mathrm{h}}=1 \mathrm{pc}$ ). Additionally, we use Equations (8) and (13) to compute the rates assuming higher-density YSCs as in the phenomenological fits of Marks \& Kroupa (2012).

We present in Table 2 the rate estimates obtained by integrating Equation (15). We find that the binary-single channel dominates over the single-single channel by a factor of roughly a few to 10 , depending upon the assumptions made
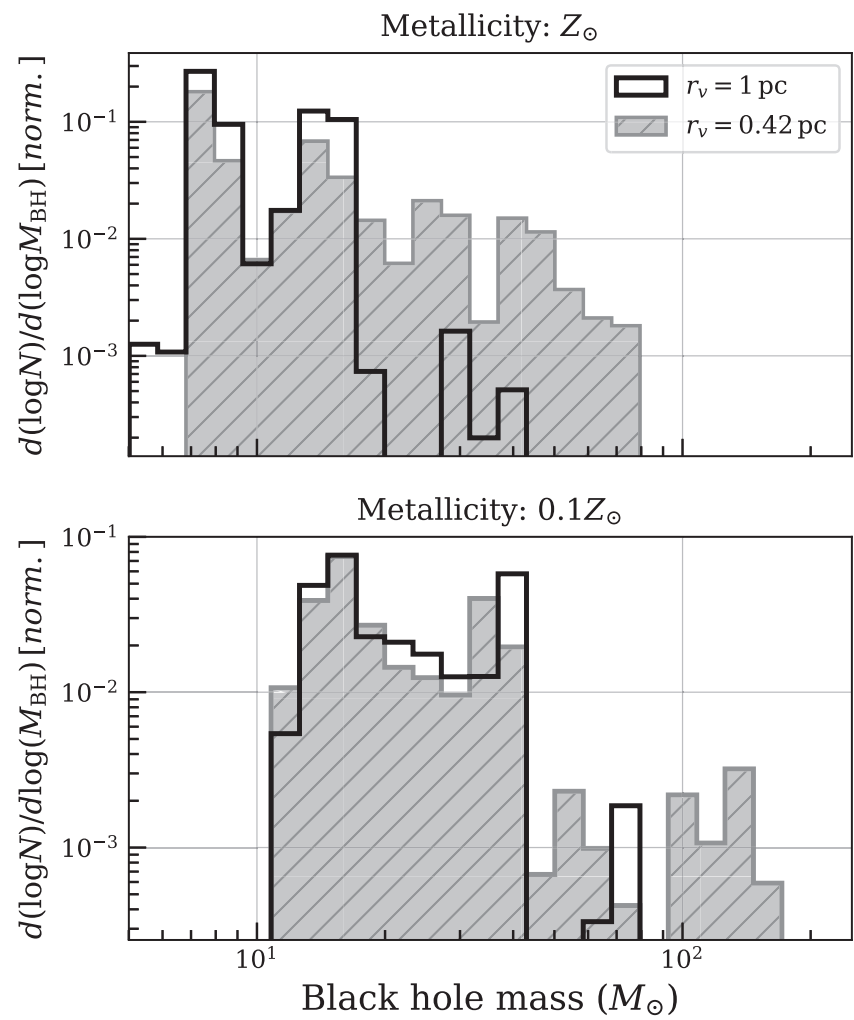

Figure 4. Normalized distribution of $\mathrm{BH}$ masses for all TDEs occurring in the various cluster models. In the top (bottom) panel, we show mass distributions for the $Z_{\odot}\left(0.1 Z_{\odot}\right)$ models. The solid black and hatched gray histograms denote models adopting a constant of $r_{v}=1 \mathrm{pc}$ and the $r_{\mathrm{h}}-M_{\mathrm{cl}}$ relation from Marks \& Kroupa (2012), respectively.

regarding $r_{\mathrm{h}}$. If we adopt the more conservative choice of constant $r_{\mathrm{h}}=1 \mathrm{pc}$, we estimate a combined TDE rate of roughly $20 \mathrm{Gpc}^{-3} \mathrm{yr}^{-1}$ in the local universe. Adopting the phenomenological assumption from Marks \& Kroupa (2012), we find a combined TDE rate of roughly $200 \mathrm{Gpc}^{-3} \mathrm{yr}^{-1}$.

For reference, Kremer et al. (2019c) predicted a TDE rate of roughly $10 \mathrm{Gpc}^{-3} \mathrm{yr}^{-1}$ for old globular clusters. In the more massive nuclear star cluster regime, Fragione et al. (2020) predicted a stellar-mass $\mathrm{BH}$ TDE rate of roughly $10^{-7}-10^{-6} \mathrm{yr}^{-1}$ per galaxy. Assuming a galactic density of roughly $10^{-2} \mathrm{Mpc}^{-3}$, this corresponds to a rate of roughly $1-10 \mathrm{Gpc}^{-3} \mathrm{yr}^{-1}$ in the local universe. Thus, we conclude YSCs may dominate the overall stellar-mass BH TDE rate in the local universe by a factor of a few to more than an order of magnitude compared to more massive clusters.

\subsection{BH Mass Function of TDEs}

In the previous subsections, we have explored specifically models a-h of Table 1, which assume solar metallicity, reflective of YSCs born recently in the local universe. However, for YSCs found at higher redshifts, assuming a lower metallicity is more appropriate. Given the overall TDE rate to be substantially higher at high redshift (see Section 3.3), a careful investigation of metallicity is warranted.

To explore the effect of cluster metallicity on TDE properties, we have run several additional sets of models with $Z=0.1 Z_{\odot}$. In Figure 4, we show the distribution of $\mathrm{BH}$ masses that undergo TDEs. The black and hatched gray histograms show $\mathrm{BH}$ masses found in models assuming $r_{\mathrm{h}}=1 \mathrm{pc}$ and the Marks \& Kroupa (2012) $r_{\mathrm{h}}-M_{\mathrm{cl}}$ relation, respectively. In the 
top (bottom) panel we show the results for models assuming solar (10\% solar) metallicity.

The first general feature we see is that higher-metallicity clusters yield lower-mass BH TDEs. This is anticipated: at higher metallicity, stellar winds are expected to lead to increased mass loss prior to stellar core collapse, which in turn is expected to reduce the mass of the $\mathrm{BH}$ ultimately formed (e.g., Vink et al. 2001; Fryer et al. 2012; Belczynski et al. 2016). For the $r_{v}=1 \mathrm{pc}$ models, we find median BH TDE masses of $13 M_{\odot}$ and $27 M_{\odot}$ for $Z_{\odot}$ and $0.1 Z_{\odot}$, respectively.

We also see that, for a given metallicity, increasing the initial cluster density yields an extended tail in the upper part of the $\mathrm{BH}$ mass spectrum. This high-mass tail is populated by $\mathrm{BHs}$ formed through stellar collisions, which occur at an increased rate in higher-density clusters. A number of recent analyses (Spera \& Mapelli 2017; Di Carlo et al. 2019a, 2019b; Kremer et al. 2020a) have demonstrated that dynamically mediated stellar collisions occurring within the first roughly $5 \mathrm{Myr}$ of cluster evolution (before formation of BHs) may lead to formation of massive stars that may ultimately collapse to form high-mass BHs. In particular, this process may permit formation of $\mathrm{BHs}$ with masses occupying the pair-instability mass gap from roughly $40-120 M_{\odot}$ expected to arise through (pulsational) pair-instability supernovae (e.g., Belczynski et al. 2016; Spera \& Mapelli 2017; Woosley 2017). Additionally, this process may be closely related to collisional runways, which have been touted as a potential formation mechanism for intermediate-mass BHs (IMBHs) with masses in excess of roughly $100 M_{\odot}$ (e.g., Portegies Zwart et al. 2004; Gürkan et al.

2006; Giersz et al. 2015; Mapelli 2016). Here, we adopt the same prescriptions implemented in Kremer et al. (2020a) to treat stellar collisions and the subsequent evolution of the collision products, as described in Section 2. We also assume the maximum $\mathrm{BH}$ mass formed through single-star evolution ( i.e., unaffected by any dynamical processes) is $40.5 M_{\odot}$, as determined by our (pulsational) pair-instability supernova treatment (see Belczynski et al. 2016, for details).

In our $Z_{\odot}$ and $0.1 Z_{\odot}$ models, which adopt the Marks \& Kroupa (2012) $r_{\mathrm{h}}$ relation, we find roughly $10 \%$ of all TDEs have $\mathrm{BH}$ masses within the assumed pair-instability gap. In the $0.1 Z_{\odot}$ models specifically, we find an additional $5 \%$ of TDEs occur with a BH mass in excess of $120 M_{\odot}$ (the assumed upper limit to the pair-instability gap). These fractions are consistent with the rates of formation of massive $\mathrm{BHs}$ shown in previous studies of YSCs (Di Carlo et al. 2019a, 2019b). Given the cosmological rates predicted in Section 3.3, these mass-gap and IMBH TDEs may constitute a non-negligible fraction of all TDEs occurring in YSCs. Furthermore, these TDEs may provide a potentially novel way to probe the formation of pairinstability gap BHs, similar to the recent $\mathrm{LIGO} / \mathrm{Virgo}$ detection GW190521 (Abbott et al. 2020a, 2020b).

Finally, comparing models $i, j, k$ with models $e, f, g$, we see that lower-metallicity clusters exhibit a moderate increase (a factor of roughly 1.4) in the total number of TDEs occurring through both single-single and binary-single encounters. This slight increase in the rate is anticipated: as shown in Equation (5), the TDE rate scales with the $\mathrm{BH}$ mass, $m_{\mathrm{BH}}$, through the influence of gravitational focusing and through tidal disruption radius calculation (Equation (1)). Thus, we expect that metallicity leads to a moderate difference in the TDE rate for a given cluster mass.

\section{EM Signatures}

In the previous section, we have shown that stellar-mass $\mathrm{BH}$ TDEs should be plentiful in YSCs. We now examine possible EM signatures of these events, building upon previous work on this subject (e.g., Perets et al. 2016; Lopez et al. 2020; Fragione et al. 2019; Kremer et al. 2019c).

\subsection{Characteristic Timescales and Luminosities}

Following the disruption of a star of mass $m_{\star}$ and radius $R_{\star}$, the timescale, $t_{\mathrm{fb}}$ for (bound) orbiting material to fallback to the disruption point, $R_{\mathrm{TD}}$ is given by

$$
\begin{aligned}
t_{\mathrm{fb}}= & \frac{\pi R_{\mathrm{TD}}^{3}}{\sqrt{2 G m_{\mathrm{BH}} R_{\star}^{3}}} \\
& \approx 1.1 \times 10^{4} \mathrm{~s}\left(\frac{m_{\star}}{M_{\odot}}\right)^{-1}\left(\frac{R_{\star}}{R_{\odot}}\right)^{3 / 2}\left(\frac{m_{\mathrm{BH}}}{10 M_{\odot}}\right)^{1 / 2}
\end{aligned}
$$

(e.g., Perets et al. 2016).

For simplicity, we assume a disk is formed promptly at radius $r_{d} \simeq R_{\mathrm{TD}}$ and take $t_{\mathrm{fb}}$ as the characteristic timescale for disk formation. This is motivated by the fact that the orbits of the bound debris are only weakly eccentric. Once a disk is formed, the timescale for the debris to accrete is set by the viscous timescale (Shakura \& Sunyaev 1973). For a thick disk, with disk height ratio $h=H / r_{d}$ (where $H$ is the disk scale height), the viscous accretion timescale is

$$
\begin{aligned}
t_{\mathrm{acc}} & \approx\left[h^{2} \alpha \Omega_{\mathrm{K}}\left(r_{d}\right)\right]^{-1} \\
& \approx 6 \times 10^{4} \mathrm{~s}\left(\frac{h}{0.5}\right)^{-2}\left(\frac{\alpha}{0.1}\right)^{-1}\left(\frac{m_{\star}}{M_{\odot}}\right)^{-1 / 2}\left(\frac{R_{\star}}{R_{\odot}}\right)^{3 / 2} .
\end{aligned}
$$

Because $t_{\mathrm{acc}}>t_{\mathrm{fb}}$, the subsequent light-curve evolution is viscosity driven (i.e., determined by accretion timescale). This marks one key difference from SMBH TDEs, where $t_{\mathrm{acc}} \ll t_{\mathrm{fb}}$ and thus, the disk evolution is likely dominated by the fallback and the accretion rate is believed to follow the standard $t^{-5 / 3}$ power law (e.g., Rees 1988; Evans \& Kochanek 1989; Phinney 1989).

For viscosity-driven accretion, assuming roughly half of the stellar material is bound to the $\mathrm{BH}$ following the TDE, the peak accretion rate can be approximated as

$$
\begin{aligned}
\dot{M}_{p} & \approx \frac{m_{\star} / 2}{t_{\mathrm{acc}}} \\
& \approx 2.6 \times 10^{2} M_{\odot} \mathrm{yr}^{-1}\left(\frac{h}{0.5}\right)^{2}\left(\frac{\alpha}{0.1}\right)\left(\frac{m_{\star}}{M_{\odot}}\right)^{3 / 2}\left(\frac{R_{\star}}{R_{\odot}}\right)^{-3 / 2} .
\end{aligned}
$$

The maximum possible luminosity is $L_{\max } \sim \epsilon \dot{M}_{p} c^{2}$, where $\epsilon \sim 0.1$ is the accretion efficiency near the innermost stable circular orbit. This case corresponds to the most efficient energy release (possibly when a jet is formed), and we estimate $L_{\max } \sim 10^{48} \mathrm{erg} \mathrm{s}^{-1}$ for typical TDE parameters. As pointed out in Perets et al. (2016), in this extreme case the TDE may power an ultra-long gamma-ray burst (e.g., Gendre et al. 2013; Levan et al. 2014).

In the more widely accepted adiabatic inflow-outflow solution model (Blandford \& Begelman 1999), the mass inflow 
of a super-Eddington disk onto a $\mathrm{BH}$ is nonconservative and only a small fraction of the mass supplied at large radii is actually accreted. The accretion rate is expected to be reduced by factor $\left(10 r_{g} / r_{d}\right)^{s}$, where $r_{g}=G m_{\mathrm{BH}} / c^{2}$ is the $\mathrm{BH}$ gravitational radius, $r_{d}$ is the disk radius, and the power-law index $s \in(0,1)$. For the most pessimistic case of $s=1$, we can estimate the accretion luminosity of the inner disk, a significant fraction of which should be observable in the X-ray band for favorable viewing angles close to face-on,

$$
\begin{aligned}
L_{\min } & \approx \epsilon\left(\frac{10 r_{g}}{r_{d}}\right) \dot{M}_{p} c^{2} \\
& \approx 1.5 \times 10^{44} \mathrm{erg} \mathrm{s}^{-1}\left(\frac{m_{\star}}{M_{\odot}}\right)^{7 / 6}\left(\frac{m_{\mathrm{BH}}}{10 M_{\odot}}\right)^{4 / 3}\left(\frac{R_{\star}}{R_{\odot}}\right)^{-5 / 2}
\end{aligned}
$$

where we have once again assumed $h=0.5$ and $\alpha=0.1$. For $0<s<1$, we expect the accretion power to be somewhere in between $10^{44}$ and $10^{48} \mathrm{erg} \mathrm{s}^{-1}$. For instance, based on numerical simulations of adiabatic accretion flows, Yuan et al. (2012) argued for $s \approx 0.5$, which corresponds to $L \sim 10^{46} \mathrm{erg} \mathrm{s}^{-1}$.

At later time $t \gg t_{\mathrm{acc}}$, the disk radius increases as $r_{d} \propto t^{2 / 3}$ due to viscous spreading, the mass inflow rate drops as $\dot{M} \propto t^{-2(s+2) / 3}$, and the $\mathrm{BH}$ accretion rate drops as $\dot{M}_{\mathrm{BH}} \propto t^{-4(s+1) / 3}$ (e.g., Kumar et al. 2008). Thus, the latetime X-ray light curve is a power law between $L_{\mathrm{X}} \propto t^{-4 / 3}$ and $t^{-8 / 3}$

The majority of the mass inflow is lost from the disk in the form of a radiatively driven wind. The energy generated by accretion in the inner disk is expected to be reprocessed by this wind and released at the photon trapping radius, $r_{\mathrm{tr}}$, where the radiative diffusion time equals the expansion time (typically occurring a few to 10 days after the TDE; Kremer et al. 2019c). A rough estimate for the trapping radius is

$$
\begin{aligned}
r_{\mathrm{tr}} \approx & \min \left(v_{\mathrm{w}} t, \frac{\dot{M}(t) \kappa}{4 \pi c}\right) \\
& \approx 10^{15} \mathrm{~cm} \min \left(\frac{v_{\mathrm{w}}}{10^{9} \mathrm{~cm} \mathrm{~s}^{-1}} \frac{t}{10^{6} \mathrm{~s}}, \frac{\dot{M}(t)}{17 M_{\odot} \mathrm{yr}^{-1}}\right),
\end{aligned}
$$

where $v_{\mathrm{w}} \approx 10^{9} \mathrm{~cm} \mathrm{~s}^{-1}$ is the typical wind speed (Kremer et al. $2019 \mathrm{c}$ ) and $\kappa=0.34 \mathrm{~cm}^{2} \mathrm{~g}^{-1}$ is the electron scattering opacity for solar composition material.

For a detailed discussion of the radiation hydrodynamics of the accretion disk and wind, we direct the reader to Kremer et al. (2019c) and Piro \& Lu (2020) and references therein. Here, we summarize the key points. As a result of adiabatic loss, the emerging luminosity is smaller than the accretion luminosity by a factor of $\left(r_{d} / r_{\mathrm{tr}}\right)^{2 / 3} \sim 10^{-2}$ for $r_{\mathrm{tr}} \sim 10^{15} \mathrm{~cm}$ and $r_{d} \sim 10^{12} \mathrm{~cm}$ roughly at $t \sim 10$ days. Kremer et al. (2019c) considered $s \in(0.2,0.8)$ and found the peak bolometric luminosity to be in the range $10^{41}-10^{44} \mathrm{erg} \mathrm{s}^{-1}$ (depending also upon the assumed stellar parameters, such as masses) and the spectrum to be in the optical/UV, for typical stellar-mass BH TDEs. This optical emission is expected to last roughly 10-100 days until the mass inflow rate drops below roughly $\left(r_{d} / r_{g}\right) L_{\mathrm{Edd}} / c^{2}$, at which point the disk is expected to transition to a geometrically thin state and the accretion rate may drop by many orders of magnitude (Shen \& Matzner 2014).
In Figure 5, we summarize the key evolutionary features of the first roughly 100 days following the tidal disruption.

\subsection{Comparison to FBOTs}

Recent high-cadence surveys have uncovered a growing number of fast-evolving transients with a wide range of observed properties. One class of particular interest is the FBOTs (Drout et al. 2014), also known as fast-evolving luminous transients (Rest et al. 2018). Although a clear understanding of FBOTs remains elusive, this class of transients is generally defined by rise times (of order one to a few days) and peak luminosities (roughly $10^{41}-10^{44} \mathrm{erg} \mathrm{s}^{-1}$ ) that are too fast and too luminous to be explained by the radioactive decay of ${ }^{56} \mathrm{Ni}$. The majority of FBOTs are found in star-forming galaxies of roughly solar metallicity. Furthermore, the explosion sites span a range of offsets from the galaxy centers, most closely resembling the offset distribution of corecollapse supernovae (e.g., Drout et al. 2014).

The majority of FBOTs have been identified via archival searches of various optical surveys, including the PanSTARRS1 Medium Deep Survey (Drout et al. 2014), the Dark Energy Survey (Pursiainen et al. 2018), Kepler (Rest et al. 2018), the Supernova Legacy Survey (Arcavi et al. 2016), and the Zwicky Transient Facility (ZTF; Ho et al. 2020). In addition to the archival searches, a handful of FBOTs have been discovered while still active, notably AT2018cow (also ATLAS18qqn; Prentice et al. 2018; Rivera Sandoval et al. 2018; Smartt et al. 2018; Margutti et al. 2019), ZTF18abvkwla (Ho et al. 2020), and CSS161010 (Coppejans et al. 2020), enabling X-ray and/or radio follow-up.

Various analyses have computed volumetric rates of transients similar to FBOTs, with estimates ranging from roughly $100 \mathrm{Gpc}^{-3} \mathrm{yr}^{-1}$ to more than $1000 \mathrm{Gpc}^{-3} \mathrm{yr}^{-1}$ in the local universe (e.g., Drout et al. 2014; Pursiainen et al. 2018; Coppejans et al. 2020; Ho et al. 2020). Although the precise rate remains uncertain, the general consensus appears to be that FBOTs are roughly two to three orders of magnitude rarer than standard core-collapse supernovae (e.g., Botticella et al. 2008).

The specific origin of FBOTs remains unknown, with a number of channels having been proposed, including TDEs by IMBHs (Perley et al. 2019), massive star collapse and BH formation (Quataert et al. 2019), electron capture collapse following a white dwarf merger (Lyutikov \& Toonen 2019), and magnetar formation (Margutti et al. 2019). Additionally, Piro \& Lu (2020) pointed out that many FBOT features (specifically in the case of AT2018cow) show similarities to what would be expected for a wind-reprocessed transient, regardless of the specific central engine.

Here, we propose stellar-mass BH TDEs as another possible FBOT progenitor. The rise times and peak optical luminosities predicted for these TDEs (Section 4.1 and Kremer et al. (2019c)) occupy the same region of parameter space expected for FBOTs, as does the estimated X-ray luminosity. ${ }^{8}$ If these TDEs occur in high-metallicity young stellar clusters (expected to be a dominant site for star formation; Lada \& Lada 2003), the host-galaxy type and offset distribution for observed FBOTs may also be recovered. Furthermore, the rate we predict for stellar-mass $\mathrm{BH}$ TDEs in YSCs (up to roughly

\footnotetext{
8 We do not consider here the variability in the X-rays observed on few day timescales in the case of AT2018cow (Rivera Sandoval et al. 2018), but note that this variability may in principle arise through orbital decay during a TDE.
} 
Tidal disruption/ Mass fallback capture

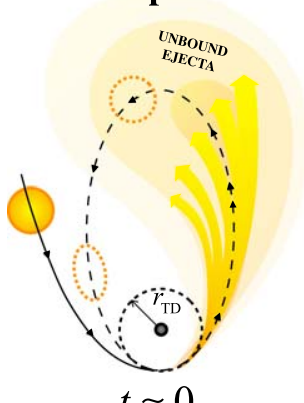

$t \approx 0$ + disk formation

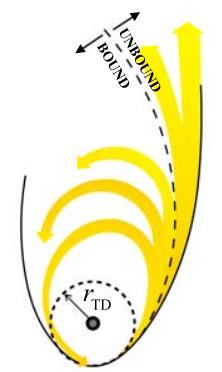

$t \approx t_{\mathrm{fb}} \approx$ hours
Accretion + wind outflow

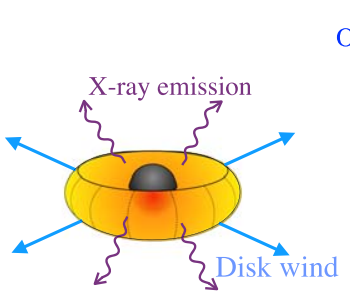

$t \approx t_{\mathrm{acc}} \approx \mathrm{day}$
Wind re-processing

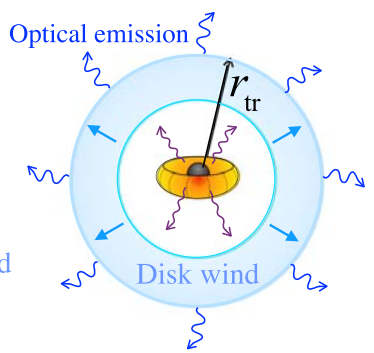

$t \approx \mathrm{a}$ few -10 days

\section{Transition to thin disk}

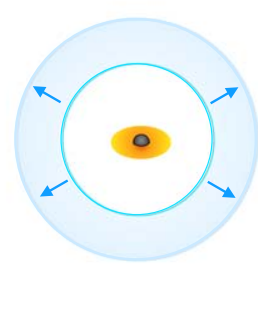

$t \approx \operatorname{month}(\mathrm{s})$

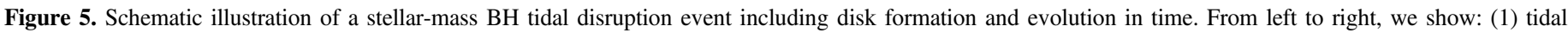

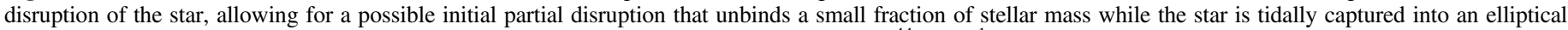

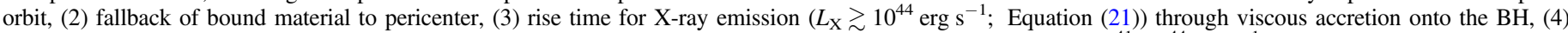

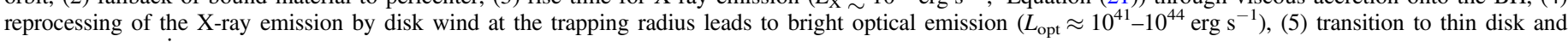
prompt drop in $\dot{M}$ and luminosity.

Table 3

Comparison of Key Features of Stellar-mass BH TDEs and FBOTs

\begin{tabular}{|c|c|c|}
\hline & Stellar-mass BH TDEs & FBOTs \\
\hline Peak optical luminosity $\left[\mathrm{erg} \mathrm{s}^{-1}\right.$ ] & $\approx 10^{41}-10^{44}$ & $\begin{array}{l}\text { Overall: } \approx 10^{41}-10^{44} \text { (Drout et al. 2014; Pursiainen et al. 2018) } \\
\text { AT2018cow: } \approx 4 \times 10^{44} \text { (Margutti et al. 2019) } \\
\text { ZTF18abvkwla: } \approx 10^{44} \text { (Ho et al. 2019) }\end{array}$ \\
\hline
\end{tabular}

Optical rise time [days] $\quad \approx$ a few -10

Overall: $\lesssim 5$ (Drout et al. 2014; Pursiainen et al. 2018)

AT2018cow: $1.43 \pm 0.08$ (Prentice et al. 2018; Perley et al. 2019) ZTF18abvkwla: $1.83 \pm 0.05$ (Ho et al. 2019)

Fade time [days] $\quad \approx$ a few

Overall: a few - 10 (Drout et al. 2014; Pursiainen et al. 2018) AT2018cow: $1.95 \pm 0.06$ (Prentice et al. 2018; Perley et al. 2019) ZTF18abvkwla: $3.12 \pm 0.22$ (Ho et al. 2019)

\begin{tabular}{|c|c|c|}
\hline $\begin{array}{l}\mathrm{X} \text {-ray luminosity }\left[\mathrm{erg} \mathrm{s}^{-1} \text { ] }\right. \\
\approx 1 \text { days after peak }\end{array}$ & $\begin{array}{l}\text { (assuming unabsorbed) } \\
\quad \approx 10^{43}-10^{47}\end{array}$ & $\begin{array}{l}\text { (Observed values are for } 0.3-10 \mathrm{keV} \text { ) } \\
\text { AT2018cow: } \approx 10^{43} \text { (Rivera Sandoval et al. 2018) }\end{array}$ \\
\hline$\approx 10$ days after peak & $\approx 10^{41}-10^{46}$ & AT2018cow: $\approx 5 \times 10^{42}$ (Rivera Sandoval et al. 2018) \\
\hline$\approx 100$ days after peak & $\approx 10^{38}-10^{45}$ & $\begin{array}{l}\text { AT2018cow: } \approx 10^{40} \text { (Margutti et al. 2019) } \\
\text { CSS161010: } \approx 5 \times 10^{39} \text { (Coppejans et al. 2020) }\end{array}$ \\
\hline Volumetric rate $\left[\mathrm{Gpc}^{-3} \mathrm{yr}^{-1}\right]$ & $20-200$ & $\begin{array}{l}<560 \text { (for } M_{g}<-20 ; \text { Ho et al. 2020) } \\
700-1400 \text { (for } M_{g}<-19 ; \text { Coppejans et al. 2020) } \\
\gtrsim 1000 \text { (for }-15.8<M_{g}<-22.2 ; \text { Pursiainen et al. 2018) }\end{array}$ \\
\hline
\end{tabular}

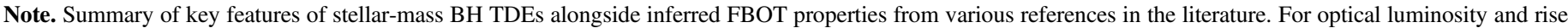

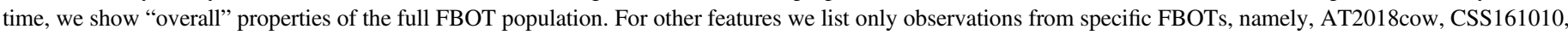

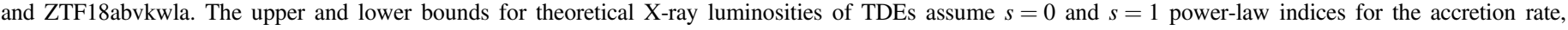
assuming no absorption; see Section 4.1.

$200 \mathrm{Gpc}^{-1} \mathrm{yr}^{-1}$ in the local universe) is comparable to the observationally inferred FBOT rates. In light of these similarities, stellar-mass BH TDEs that occur in YSCs may in principle be a viable progenitor for FBOT-like transients. In Table 3, we summarize for comparison the key features of both FBOTs and stellar-mass BH TDEs.

Although it remains to be determined whether bright radio emission is a defining feature of the FBOT class broadly, radio emission (consistent with self-absorbed synchrotron radiation) is observed for the AT2018cow, ZTF18abvkwla, and CSS161010 events. This radio emission suggests the presence of circumstellar medium with densities ranging from roughly $10-10^{6} \mathrm{~cm}^{-3}$, for various observation epochs and for a range of microphysics assumptions (Margutti et al. 2019; Coppejans et al. 2020; Ho et al. 2020). If prior to its tidal disruption, a main-sequence star is tidally captured by a $\mathrm{BH}$, such that a small amount of orbital energy is deposited into the star (e.g., Fabian et al. 1975, see left-most panel of Figure 5 for illustration), a small amount of debris may be unbound from the star. This may occur either through partial stripping by the $\mathrm{BH}$ at the first pericenter passage or during subsequent pericenter passages if the star's envelope expands due to energy injected through the tidal encounter. The circumstellar medium density inferred from radio emission may be produced by a small amount of ejecta $\left(\lesssim 10^{-2} M_{\odot}\right)$ homologously expanding for a few years at a speed of $10^{3} \mathrm{~km} \mathrm{~s}^{-1}$. More 
detailed hydrodynamic models of the disruption process are required to calculate the radial density profile and predict the radio emission.

\section{Conclusions and Discussion}

\subsection{Summary}

We summarize here the main findings of this work:

1. Using a suite of roughly $3000 \mathrm{~N}$-body simulations, we have explored the rates and properties of stellar-mass $\mathrm{BH}$ TDEs in YSCs. We derived TDE rates as a function of cluster mass and showed that the rates derived from the models agree closely with rates derived from simple analytic estimates.

2. Using the rate scalings derived from our models and integrating over the full cluster mass function, we predict these TDEs occur at a rate of roughly $20-200 \mathrm{Gpc}^{-3} \mathrm{yr}^{-1}$ in the local universe. The range in this estimate is determined primarily by the assumptions made concerning initial cluster densities (e.g., Portegies Zwart et al. 2010; Marks \& Kroupa 2012). Overall, we find TDEs occur a factor of a few to 10 times more frequently in binary-mediated dynamical encounters compared to single-single encounters.

3. In YSCs of roughly solar metallicity, we predict a median BH mass of roughly $10 M_{\odot}$. In lower-metallicity $(Z \lesssim 0.1$ $Z_{\odot}$ ) clusters that may have formed at higher redshift, we predict a median mass of roughly $30 M_{\odot}$. This difference is primarily a result of the metallicity-dependent stellar wind mass loss (Vink et al. 2001).

4. We showed that stellar-mass BH TDEs exhibit the following key features: (i) rise times of roughly a day driven by the viscous accretion timescale, (ii) peak X-ray luminosities of roughly $10^{44}-10^{48} \mathrm{erg} \mathrm{s}^{-1}$, and (iii) optical luminosities of up to roughly $10^{44} \mathrm{erg} \mathrm{s}^{-1}$ produced by reprocessing of X-rays by a disk wind on a timescale of a few to 10 days after the TDE. On the basis of all of these features, combined with the estimated TDE rates and host-galaxy properties, we propose stellarmass BH TDEs as a viable progenitor for the FBOT class of transients (e.g., Drout et al. 2014; Margutti et al. 2019; Coppejans et al. 2020; Ho et al. 2020).

\subsection{Discussion and Future Work}

Stellar-mass BH TDEs distinguish themselves from other proposed channels for FBOTs (see Section 4.2) in the combination of X-ray and optical signatures. For instance, in the case of stellar collapse, long-lasting power from a central engine may be supplied by fallback accretion onto a $\mathrm{BH}$ or magnetar spindown, where the late-time energy injection rates are expected to be $L \propto t^{-5 / 3}$ and $L \propto t^{-2}$, respectively. In our model for stellar-mass BH TDEs, the energy injection rate may have a broad range of energy injection rates from $L \propto t^{-4 / 3}-L \propto t^{-8 / 3}$. This leads to different late-time $(t \gtrsim 10$ days) behaviors in the light curve that can in principle to used to distinguish our model from others. Of course, the detection of a host YSC may also hint at the proposed TDE scenario (for example, see Lyman et al. 2020, for a discussion of host features of AT2018cow). However, core-collapse supernovae will also occur in sufficiently YSCs, thus, the presence of a host cluster may not provide indisputable evidence of a TDE origin.

Although the event rates inferred from FBOT observations are uncertain and vary between different analyses, in general, the observed FBOT rate appears higher than the stellar-mass $\mathrm{BH}$ TDE rate estimated from our models by a small factor (see Table 3). However, there are several reasons why our estimated rate may underestimate the true TDE rate, possibly explaining this discrepancy:

First, in this study, we have assumed zero primordial binaries. BH binaries were allowed to form exclusively through three-body formation (see Section 2). Stellar binaries are known to increase the rates of dynamical collisions and TDEs (e.g., Fregeau \& Rasio 2007). In this case, our predicted TDE rates may be a lower limit. To test this, we ran 50 additional simulations ( $O$ in Table 1 ) that adopt a primordial binary fraction of $100 \%$. Consistent with previous CMC studies (e.g., Kremer et al. 2020b), secondary masses are drawn from a uniform distribution in mass ratio in the range of $[0.1,1]$ and initial orbital periods are drawn from a log-uniform distribution (e.g., Sana et al. 2012). In total, these models yield a factor of roughly 5 more TDEs per cluster (all of which occur through binary-mediated encounters) compared to the models with comparable mass and zero primordial binaries ( $f$ in Table 1). Thus, primordial binaries may increase the TDE rates quoted in Table 2 by a small factor. We reserve for follow-up studies a more expansive investigation of primordial binaries and their implications for binary-mediated TDEs.

Second, as discussed briefly in Section 4, a fraction of TDEs may occur through tidal capture where the main-sequence star may undergo multiple passages before ultimately being disrupted. As discussed in, e.g., Fabian et al. (1975), the cross section for tidal capture may be a factor of roughly a few times larger than that for tidal disruption. If the fate of the majority of tidal captures is a TDE, the total TDE rate may be higher than that estimated here by a factor of a few.

Of course, realistic YSCs exhibit a much larger range in properties than those considered here. For instance, in our $\mathrm{N}$ body models, we have adopted a relatively narrow range in initial cluster sizes, which may in reality extend from roughly $0.1-10 \mathrm{pc}$ or even more, depending on the cluster mass (e.g., Krumholz et al. 2019). Although our models were intended to capture the "mean" of this distribution, this value is uncertain, and of course, lower-density clusters would exhibit a lower TDE rate. For example, from the supplemental models $n$ in Table 1, we find that the TDE rate for clusters with initial $r_{v}=2 \mathrm{pc}$ is a factor of roughly 6 times lower than than the rates computed in the $r_{v}=1 \mathrm{pc}$ models. If indeed $r_{v}=2 \mathrm{pc}$ is more representative of the full distribution of YSCs, the volumetric rates shown in Table 2 may overestimate the true value.

Along similar lines, we have adopted a relatively narrow range in initial cluster masses that does not span the full distribution of observed YSCs. For example, YSCs in the antennae are observed with masses of $10^{6} M_{\odot}$ (e.g., Portegies Zwart et al. 2010) or more, while observed clusters in massive elliptical galaxies can reach $10^{7} M_{\odot}$ (e.g., Bastian et al. 2006). Although of high potential interest (see, e.g., Rodriguez et al. 2020 , for a recent study of super-star clusters with CMC), we reserve consideration of these high-mass systems for later study.

In Section 3.4, we showed that, depending on the assumed initial virial radius and cluster metallicity, IMBHs with masses 
in excess of $100 M_{\odot}$ may form in YSCs through stellar collisions (see also Di Carlo et al. 2019a; Kremer et al. 2020a) and ultimately undergo TDEs. The topic of TDEs by IMBHs in stellar clusters has been examined at length (e.g., Rosswog et al. 2009; MacLeod et al. 2014, 2016; Fragione et al. 2020). We have focused in this study on the more common (by a factor of roughly 10:1 or higher in our models) stellar-mass $\mathrm{BH}$ TDE. However, IMBH TDEs likely lead to several notable differences. For example, for sufficiently massive IMBHs, the TDEs will transition from viscosity driven to fallback driven, thus becoming qualitatively more similar to an SMBH TDE (e.g., Rees 1988). We leave for future work a more careful examination of the properties and observational signatures of TDEs by IMBHs.

We have used here the $\mathrm{CMC}$ code to model the evolution of YSCs. The Monte Carlo-based approach used in CMC allows us to model large populations of clusters at low computational expense compared to direct $N$-body models (e.g., Pattabiraman et al. 2013; Rodriguez et al. 2016). However, unlike massive globular clusters, the relatively low-mass YSCs studied here can also be studied efficiently with direct $N$-body models (e.g., Banerjee 2017; Di Carlo et al. 2019a). Future work may examine the topic of TDEs using direct $N$-body models, which would allow detailed examination of several regimes that Monte Carlo simulations like CMC are ill-equipped to study. For example, examination of the final stage of cluster's life as it dissolves on a dynamical timescale through its tidal boundary and examination of clusters that contain massive IMBHs that may affect the overall cluster and TDE dynamics. Additionally, direct $N$-body models are more suited to study the lowest-mass stellar associations $(N \lesssim 100$; Lada \& Lada 2003), for which the spherical symmetry assumptions at the heart of the Monte Carlo-based approach break down.

Finally, unlike SMBH TDEs, which have been studied extensively with hydrodynamic simulations, the stellar-mass $\mathrm{BH}$ regime has been little explored, with a few exceptions (Perets et al. 2016; Lopez et al. 2020). Ultimately, hydrodynamic models are necessary to understand the detailed features of these events. For example, in the analytic estimates in Section 4, we considered only those interactions near the tidal disruption boundary, $r_{\mathrm{TD}}$, of the star. However, given that the interaction cross section scales linearly with stellar radius (Equation (4)), a fraction of such BH-star encounters may actually occur in the direct collision regime, where $r_{p}<R_{\star}$ (e.g., Fryer \& Woosley 1998; Hansen \& Murali 1998). Although this subclass of "head-on" encounters is unlikely to affect our rate predictions by more than a small factor, these physical collisions may produce a subclass of unique transients. For instance, a direct collision may lead to prompt accretion ( i.e., $t_{\mathrm{fb}} \approx 0$ ) onto the $\mathrm{BH}$, which, among other possible consequences, may make the effects of feedback critical on the subsequent evolution, if indeed a disk forms at all. On the other hand, as mentioned briefly in Section 4, even more distant encounters $\left(r_{p}>r_{\mathrm{TD}}\right)$ may lead to tidal capture, possibly resulting in multiple passages that each partially strip the star (e.g., Fabian et al. 1975; Ivanova et al. 2017), again potentially producing EM signatures unique from those presented in the classic TDE regime discussed in Section 4. More careful treatment of the various regimes of $\mathrm{BH}-$ star interactions with hydrodynamic models is necessary to explore the potentially broad range of outcomes of these events in greater detail.
We thank Tom Maccarone for helpful suggestions on the manuscript and Carl Rodriguez for useful preliminary discussions. We also thank the anonymous referee for the careful review and many helpful suggestions. K.K. is supported by an NSF Astronomy and Astrophysics Postdoctoral Fellowship under award AST-2001751. W.L. is supported by the David and Ellen Lee Fellowship at Caltech. S.C. acknowledges support of the Department of Atomic Energy, Government of India, under project no. 12-R\&D-TFR-5.02-0200. F.A.R. and C.S.Y. acknowledge support from NSF grant AST-1716762 at Northwestern University.

\section{ORCID iDs}

Kyle Kremer (iD https://orcid.org/0000-0002-4086-3180 Wenbin Lu (iD https://orcid.org/0000-0002-1568-7461 Anthony L. Piro (iD https://orcid.org/0000-0001-6806-0673 Sourav Chatterjee (iD https://orcid.org/0000-0002-3680-2684 Frederic A. Rasio (iD https://orcid.org/0000-0002-7132-418X Claire S. Ye (iD https://orcid.org/0000-0001-9582-881X

\section{References}

Abbott, R., Abbott, T. D., Abraham, S., et al. 2020a, ApJL, 900, L13 Abbott, R., Abbott, T. D., Abraham, S., et al. 2020b, PhRvL, 125, 101102 Antonini, F., \& Gieles, M. 2020, MNRAS, 492, 2936

Arca Sedda, M., Askar, A., \& Giersz, M. 2018, MNRAS, 479, 4652

Arcavi, I., Wolf, W. M., Howell, D. A., et al. 2016, ApJ, 819, 35

Askar, A., Arca Sedda, M., \& Giersz, M. 2018, MNRAS, 478, 1844 Banerjee, S. 2017, MNRAS, 467, 524

Banerjee, S. 2018, MNRAS, 481, 5123

Banerjee, S. 2021, MNRAS, 500, 3002

Bastian, N., Saglia, R. P., Goudfrooij, P., et al. 2006, A\&A, 448, 881 Bate, M. R., Bonnell, I. A., \& Bromm, V. 2003, MNRAS, 339, 577 Belczynski, K., Heger, A., Gladysz, W., et al. 2016, A\&A, 594, A97 Bellm, E. C., Kulkarni, S. R., Graham, M. J., et al. 2019, PASP, 131, 018002 Blandford, R. D., \& Begelman, M. C. 1999, MNRAS, 303, L1

Botticella, M. T., Riello, M., Cappellaro, E., et al. 2008, A\&A, 479, 49 Breen, P. G., \& Heggie, D. C. 2013, MNRAS, 432, 2779

Carpenter, J. M. 2000, AJ, 120, 3139

Chambers, K. C., Magnier, E. A., Metcalfe, N., et al. 2016, arXiv:1612.05560

Chatterjee, S., Rodriguez, C. L., \& Rasio, F. A. 2017, ApJ, 834, 68

Chomiuk, L., Strader, J., Maccarone, T. J., et al. 2013, ApJ, 777, 69

Coppejans, D. L., Margutti, R., Terreran, G., et al. 2020, ApJL, 895, L23

Coughlin, E. R., Armitage, P. J., Nixon, C., \& Begelman, M. C. 2017 MNRAS, 465, 3840

Darbha, S., Coughlin, E. R., Kasen, D., \& Quataert, E. 2018, MNRAS, 477, 4009

Di Carlo, U. N., Giacobbo, N., Mapelli, M., et al. 2019a, MNRAS, 487, 2947 Di Carlo, U. N., Mapelli, M., Bouffanais, Y., et al. 2019b, MNRAS, 497, 1043 Drout, M. R., Chornock, R., Soderberg, A. M., et al. 2014, ApJ, 794, 23

Evans, C. R., \& Kochanek, C. S. 1989, ApJL, 346, L13

Fabian, A. C., Pringle, J. E., \& Rees, M. J. 1975, MNRAS, 172, 15p

Fragione, G., Leigh, N. W. C., Perna, R., \& Kocsis, B. 2019, MNRAS, 489, 727

Fragione, G., Perna, R., \& Loeb, A. 2021, MNRAS, 500, 4307

Fregeau, J. M., \& Rasio, F. A. 2007, ApJ, 658, 1047

Fryer, C. L., Belczynski, K., Wiktorowicz, G., et al. 2012, ApJ, 749, 91

Fryer, C. L., \& Woosley, S. E. 1998, ApJL, 502, L9

Gendre, B., Stratta, G., Atteia, J. L., et al. 2013, ApJ, 766, 30

Gieles, M., Portegies Zwart, S. F., Baumgardt, H., et al. 2006, MNRAS, 371,793

Giersz, M., Askar, A., Wang, L., et al. 2019, MNRAS, 487, 2412

Giersz, M., Leigh, N., Hypki, A., Lützgendorf, N., \& Askar, A. 2015, MNRAS, 454, 3150

Giesers, B., Dreizler, S., Husser, T.-O., et al. 2018, MNRAS, 475, L15

Giesers, B., Kamann, S., Dreizler, S., et al. 2019, A\&A, 632, A3

Giesler, M., Clausen, D., \& Ott, C. D. 2018, MNRAS, 477, 1853

Gürkan, M. A., Fregeau, J. M., \& Rasio, F. A. 2006, ApJL, 640, L39

Hansen, B. M. S., \& Murali, C. 1998, ApJL, 505, L15 
Heggie, D., \& Hut, P. 2003, The Gravitational Million-Body Problem: A Multidisciplinary Approach to Star Cluster Dynamics (Cambridge: Cambridge Univ. Press)

Ho, A. Y. Q., Perley, D. A., Kulkarni, S. R., et al. 2020, ApJ, 895, 49

Ho, A. Y. Q., Phinney, E. S., Ravi, V., et al. 2019, ApJ, 871, 73

Hopkins, A. M., \& Beacom, J. F. 2006, ApJ, 651, 142

Hurley, J. R., Pols, O. R., \& Tout, C. A. 2000, MNRAS, 315, 543

Hurley, J. R., Tout, C. A., \& Pols, O. R. 2002, MNRAS, 329, 897

Ivanova, N., Chaichenets, S., Fregeau, J., et al. 2010, ApJ, 717, 948

Ivanova, N., da Rocha, C. A., Van, K. X., \& Nandez, J. L. A. 2017, ApJL, 843, L 30

Joshi, K. J., Rasio, F. A., Zwart, S. P., \& Portegies Zwart, S. 2000, ApJ, 540, 969

King, I. 1962, AJ, 67, 471

Kremer, K., Chatterjee, S., Rodriguez, C. L., \& Rasio, F. A. 2018a, ApJ, 852,29

Kremer, K., Chatterjee, S., Ye, C. S., Rodriguez, C. L., \& Rasio, F. A. 2019a, ApJ, 871, 38

Kremer, K., D’Orazio, D. J., Samsing, J., Chatterjee, S., \& Rasio, F. A. 2019b, ApJ, 885, 2

Kremer, K., Lu, W., Rodriguez, C. L., Lachat, M., \& Rasio, F. 2019c, ApJ, 881,75

Kremer, K., Spera, M., Becker, D., et al. 2020a, ApJ, 903, 45

Kremer, K., Ye, C. S., Chatterjee, S., Rodriguez, C. L., \& Rasio, F. A. 2018b, ApJL, 855, L15

Kremer, K., Ye, C. S., Rui, N. Z., et al. 2020b, ApJS, 247, 48

Kroupa, P. 2001, MNRAS, 322, 231

Kruijssen, J. M. D., Pelupessy, F. I., Lamers, H. J. G. L. M., Portegies Zwart, S. F., \& Icke, V. 2011, MNRAS, 414, 1339

Krumholz, M. R., McKee, C. F., \& Bland-Hawthorn, J. 2019, ARA\&A, 57,227

Kulkarni, S. R., Hut, P., \& McMillan, S. 1993, Natur, 364, 421

Kumar, P., Narayan, R., \& Johnson, J. L. 2008, MNRAS, 388, 1729

Lada, C. J., \& Lada, E. A. 2003, ARA\&A, 41, 57

Lamers, H. J. G. L. M., Gieles, M., \& Portegies Zwart, S. F. 2005, A\&A, 429,173

Law, N. M., Kulkarni, S. R., Dekany, R. G., et al. 2009, PASP, 121, 1395

Levan, A. J., Tanvir, N. R., Starling, R. L. C., et al. 2014, ApJ, 781, 13

Liu, F. K., Li, S., \& Chen, X. 2009, ApJL, 706, L133

Liu, F. K., Li, S., \& Komossa, S. 2014, ApJ, 786, 103

Lopez, M., Jr., Batta, A., Ramirez-Ruiz, E., Martinez, I., \& Samsing, J. 2020 , ApJ, 903, 45

LSST Science Collaboration, Abell, P. A., Allison, J., et al. 2009, arXiv:0912.0201

Lyman, J. D., Galbany, L., Sánchez, S. F., et al. 2020, MNRAS, 495, 992

Lyutikov, M., \& Toonen, S. 2019, MNRAS, 487, 5618

Maccarone, T. J., Kundu, A., Zepf, S. E., \& Rhode, K. L. 2007, Natur, 445,183

Mackey, A. D., Wilkinson, M. I., Davies, M. B., \& Gilmore, G. F. 2007, MNRAS, 379, L40

MacLeod, M., Goldstein, J., Ramirez-Ruiz, E., Guillochon, J., \& Samsing, J. 2014, ApJ, 794, 9

MacLeod, M., Guillochon, J., Ramirez-Ruiz, E., Kasen, D., \& Rosswog, S. 2016, ApJ, 819, 3

Mapelli, M. 2016, MNRAS, 459, 3432

Margutti, R., Metzger, B. D., Chornock, R., et al. 2019, ApJ, 872, 18
Marks, M., \& Kroupa, P. 2012, A\&A, 543, A8

Miller-Jones, J. C. A., Strader, J., Heinke, C. O., et al. 2015, MNRAS, 453, 3918

Morscher, M., Pattabiraman, B., Rodriguez, C., Rasio, F. A., \& Umbreit, S. 2015, ApJ, 800, 9

Pattabiraman, B., Umbreit, S., Liao, W.-k., et al. 2013, ApJS, 204, 15

Perets, H. B., Li, Z., Lombardi, J. C., Jr., \& Milcarek, S. R., Jr. 2016, ApJ, 823, 113

Perley, D. A., Mazzali, P. A., Yan, L., et al. 2019, MNRAS, 484, 1031

Phinney, E. S. 1989, in The Center of the Galaxy, ed. M. Morris, Vol. 136 (Dordrecht: Kluwer Academic ), 543

Piro, A. L., \& Lu, W. 2020, ApJ, 894, 2

Portegies Zwart, S. F., Baumgardt, H., Hut, P., Makino, J., \& McMillan, S. L. W. 2004, Natur, 428, 724

Portegies Zwart, S. F., McMillan, S. L. W., \& Gieles, M. 2010, ARA\&A, 48, 431

Prentice, S. J., Maguire, K., Smartt, S. J., et al. 2018, ApJL, 865, L3

Pursiainen, M., Childress, M., Smith, M., et al. 2018, MNRAS, 481, 894

Quataert, E., Lecoanet, D., \& Coughlin, E. R. 2019, MNRAS, 485, L83

Rees, M. J. 1988, Natur, 333, 523

Rest, A., Garnavich, P. M., Khatami, D., et al. 2018, NatAs, 2, 307

Rivera Sandoval, L. E., Maccarone, T. J., Corsi, A., et al. 2018, MNRAS, 480, L146

Rodriguez, C. L., Amaro-Seoane, P., Chatterjee, S., \& Rasio, F. A. 2018 , PhRvL, 120, 151101

Rodriguez, C. L., Chatterjee, S., \& Rasio, F. A. 2016, PhRvD, 93, 084029

Rodriguez, C. L., Kremer, K., Grudić, M. Y., et al. 2020, ApJL, 896, L10

Rodriguez, C. L., \& Loeb, A. 2018, ApJL, 866, L5

Rodriguez, C. L., Morscher, M., Wang, L., et al. 2016, MNRAS, 463, 2109

Rosswog, S., Ramirez-Ruiz, E., \& Hix, W. R. 2009, ApJ, 695, 404

Samsing, J. 2018, PhRvD, 97, 103014

Samsing, J., MacLeod, M., \& Ramirez-Ruiz, E. 2017, ApJ, 846, 36

Samsing, J., Venumadhav, T., Dai, L., et al. 2019, PhRvD, 100, 043009

Sana, H., de Mink, S. E., de Koter, A., et al. 2012, Sci, 337, 444

Shakura, N. I., \& Sunyaev, R. A. 1973, A\&A, 500, 33

Shappee, B. J., Prieto, J. L., Grupe, D., et al. 2014, ApJ, 788, 48

Shen, R.-F., \& Matzner, C. D. 2014, ApJ, 784, 87

Shishkovsky, L., Strader, J., Chomiuk, L., et al. 2018, ApJ, 855, 55

Sigurdsson, S. 1993, ApJL, 415, L43

Smartt, S. J., Clark, P., Smith, K. W., et al. 2018, ATel, 11727, 1

Spera, M., \& Mapelli, M. 2017, MNRAS, 470, 4739

Strader, J., Chomiuk, L., Maccarone, T. J., Miller-Jones, J. C. A., \& Seth, A. C. 2012, Natur, 490, 71

Tonry, J. L., Denneau, L., Heinze, A. N., et al. 2018, PASP, 130, 064505

Vink, J. S., de Koter, A., \& Lamers, H. J. G. L. M. 2001, A\&A, 369, 574

Wang, L. 2020, MNRAS, 491, 2413

Wang, L., Spurzem, R., Aarseth, S., et al. 2015, MNRAS, 450, 4070

Weatherford, N. C., Chatterjee, S., Kremer, K., \& Rasio, F. A. 2020, ApJ, 898,162

Woosley, S. E. 2017, ApJ, 836, 244

Ye, C. S., Kremer, K., Chatterjee, S., Rodriguez, C. L., \& Rasio, F. A. 2019, ApJ, 877, 122

Yuan, F., Wu, M., \& Bu, D. 2012, ApJ, 761, 129

Ziosi, B. M., Mapelli, M., Branchesi, M., \& Tormen, G. 2014, MNRAS, 441,3703 